\title{
Efficacy and safety of various anti-rheumatic treatments for patients with rheumatoid arthritis: a network meta-analysis
}

\author{
Kexun Ma ${ }^{1}$, Ling $\mathrm{Li}^{2}$, Chunhui Liư ${ }^{1}$, Lingling Zhou ${ }^{3}$, Xueping Zhou ${ }^{1}$
}

${ }^{1}$ The First Clinical College, Nanjing University of Chinese Medicine, Nanjing, Jiangsu, China

2Department of Rheumatology, Taizhou Hospital of TCM, Taizhou, Jiangsu, China

${ }^{3}$ College of Pharmacy, Nanjing University of Chinese Medicine, Nanjing, Jiangsu, China

Submitted: 23 December 2016

Accepted: 22 March 2017

Arch Med Sci 2019; 15, 1: 33-54

DOI: https://doi.org/10.5114/aoms.2018.73714

Copyright $\odot 2018$ Termedia \& Banach

\section{Abstract}

Introduction: Biologics and traditional disease-modifying anti-rheumatic drugs (DMARDs) are generally used in treating patients with rheumatoid arthritis (RA). Previous studies have presented abundant data and information about the efficacy of such treatments, but the results were incomplete and inconclusive. This network meta-analysis was conducted to compare and assess the efficacy and safety of 15 therapies employing biologics and DMARDs for RA patients.

Material and methods: Six outcomes (American College of Rheumatology $20 \%$ response rate (ACR20), ACR50, ACR70, remission, adverse events (AEs) and serious adverse events (SAEs)) were used to evaluate the efficacy and safety of different treatments. The node-splitting method was used to assess the inconsistency, and the rank probabilities of the therapies were estimated by surface under the cumulative ranking curve. Besides, Jadad scale was used to evaluate the methodological quality of eligible studies.

Results: A total of 67 randomized controlled trials with 20,898 patients met the inclusion criteria. Most of the therapies presented better performance than conventional DMARDs (CDMARDs) and placebo in ACR20, ACR50 and ACR70. Conversely, the safety of CDMARDs and placebo seemed to be superior in AEs and SAEs. Also, tocilizumab (TCZ) and TCZ + methotrexate (MTX) showed better remission in pain compared to other treatments. Overall, certolizumab pegol (CZP) + MTX and TCZ + MTX had higher probability than the other treatments in efficacy outcomes.

Conclusions: We recommend CZP + MTX as the optimal drug therapy because it has the highest ranking in efficacy outcomes and relatively low risk of adverse events. TCZ + MTX is recommended as an alternative. Abatacept $(A B T)$ and $c D M A R D s$ are not recommended due to their low efficacy.

Key words: rheumatoid arthritis, biologics, disease-modifying antirheumatic drugs, network meta-analysis.

\section{Introduction}

Rheumatoid arthritis (RA), a chronic systemic autoimmune disease distributed in all racial and ethnic groups, leads to joint stiffness, deformity and damage [1]. It is characterized by irreversible, alternating episodes, swelling, pain and tenderness, which results in worsening

\section{Corresponding author:}

Xueping Zhou

The First Clinical College

Nanjing University of Chinese Medicine

No. 138 Xianlin Avenue

Qixia District, Nanjing 210023

Jiangsu, China

Phone: +8602585811642

E-mail: yuhuali05@163.com 
of physical condition, a reduction of life quality, a decline in employment and increasing direct or indirect expenses [2]. Based on recent statistics, the morbidity of patients with RA in developed countries was approximately $1 \%$ in the adult population [3]. Generally, the prevalence of RA in Asian countries was less than that in North America (1.1-1.6\%) or in Northern Europe (0.4-0.8\%) [4].

A number of drugs which were used in treating the patients with RA separately or together responded well. Among them, infliximab, etanercept, adalimumab, golimumab, tocilizumab, abatacept, certolizumab pegol, methotrexate, and conventional disease-modifying anti-rheumatic drugs (cDMARDs) were the most common choices [5]. A report showed that the difference between certolizumab pegol and placebo in the American College of Rheumatology 20\% response rate (ACR20) was statistically significant from 1 week to 24 weeks. For example, the ACR20 was $45.5 \%$ for certolizumab pegol (400 mg every 4 weeks) compared to $9.3 \%$ for placebo at week 24 [6]. However, in order to minimize the risk of neutralizing antibodies and to enhance efficacy, biologic agents are combined with cDMARDs most of the time, though several biologic agents were applied as single therapy [7]. According to the studies, patients with RA treated with placebo plus methotrexate, golimumab $(100 \mathrm{mg}$ ) plus placebo, golimumab $(50 \mathrm{mg})$ plus methotrexate and golimumab (100 mg) plus methotrexate had ACR20 response rates of $33.1 \%$, 44.4\%, 55.1\% and 56.2\% respectively. Apparently, the therapy combining golimumab with methotrexate can significantly relieve the disease and improve the physical condition [8].

Up to now, there have been dozens of pair-wise meta-analyses (MA) and network meta-analyses (NMA) which evaluate the efficacy and safety of different drug therapies for patients with RA. Nevertheless, most of the trials only focused on two interventions or just a few kinds of drugs, and some of the initial MAs were contradicted by subsequent studies. For instance, a 55\% increase in risk of serious infection for patients who were treated with biologics was reported by a Cochrane review [9], while another trial evaluating malignancy risk in RA patients concluded that there was no significant evidence of an increased risk of malignancy using biologics [10]. In contrast, Bongartz et al. reported that RA patients who were treated by anti-TNF therapies had an increased risk of serious infections and malignancies [11]. Therefore, although previous studies have shown abundant data and information, they just verified the efficacy or safety of various therapies for patients with RA. However, the lack of head-to-head comparisons and the absence of systematical comparison made the results incomplete and inconclusive.
An NMA seeks to infer the relative efficacy of two treatments by direct and indirect comparisons. Simultaneously, it extracts and analyzes data from all randomized control trials (RCTs) to select the best therapy [12]. Four efficacy outcomes and two safety outcomes were chosen to systematically assess 15 therapies from 56 RCTs with a sample size of 20,898 patients. The objective of the current study is to better characterize the efficacy and safety of each treatment for patients with RA and then make the best choice in clinical practice.

\section{Material and methods}

\section{Search strategy}

We performed a systematic literature search in electronic databases, including PubMed, Embase and Cochrane Library, to retrieve eligible RCTs from 1997 to 2016. Key words and subject terms included "rheumatoid arthritis", "biological factors", "anti-TNF agents", "infliximab", "etanercept", "adalimumab", "golimumab", "certolizumab pegol", and "rituximab". Two reviewers performed the initial search, and all references were reviewed to identify additional studies that were not included in the retrieval. After that, they screened the titles and abstracts to make sure that the studies met predefined selection criteria individually.

\section{Inclusion and exclusion criteria}

Studies included should meet the following criteria: (1) the study design should be RCT; (2) the trials included at least one pairwise comparison between two interventions, which should be used to treat patients with RA; (3) detailed data of at least one relevant outcome were provided. In addition, we excluded duplicate data, reviews, meeting or conference abstracts and case reports from the current analysis.

\section{Outcome measurement and data extraction}

The information as follows was extracted from each eligible study: study code, first author, year of publication, country in which the study was conducted, length of follow-up, interventions, sample size of each therapy and respective outcomes for efficacy and safety. There were 6 outcome indicators to assess the efficacy and safety. American College of Rheumatology 20\%, 50\%, and $70 \%$ response rate (ACR20, ACR50 and ACR70, defined as a 20\%, 50\% and $70 \%$ improvement in patients) at 12-54 weeks and remission were the efficacy outcomes. Among them, the primary efficacy endpoints were ACR20 and ACR50, and the 
secondary endpoints were ACR70 and remission. Meanwhile, adverse events (AEs) and serious adverse events (SAEs) were safety outcomes.

\section{Statistical analysis}

The indirect and direct evidence from a wide range of data was analyzed through a Bayesian NMA. After each pair-wise comparison was conducted, the network diagrams of ACR20, ACR50, $A C R 70$, remission, $A E s$ and SAEs were plotted with different interventions. The size of circles indicated the quantity of specific interventions and the boldness of arms showed the number of included studies. The results of these binary variables were presented as odds ratios (ORs) with corresponding 95\% credible intervals (Crls). In addition, net heat plots and node-splitting test were used to analyze the inconsistency level between indirect and direct evidence. The rank probabilities of efficacy and safety of 15 therapies were assessed using surface under the cumulative ranking curve (SUCRA), and the Jadad scale was used to evaluate the methodological quality of eligible studies. All statistical analyses were implemented by STATA version 12.0.

\section{Results}

\section{Studies included in the network} meta-analysis

According to a systematic literature search in electronic databases, a total of 8,104 records were identified. Among them, 1,465 duplicate publications and 6,394 articles were excluded due to their irrelevant titles and abstracts. The remaining 245 articles were selected for full-text review and 178 articles assessed as ineligible were excluded. Eventually, 67 RCTs dating from 1997 to 2016 met the inclusion criteria with 20,898 patients [13-79]. The searching and selection steps are shown in Figure 1.

\section{Characteristics of included studies}

The characteristics of included trials are shown in Table I. In detail, 33 of 56 different trials covered patients around the world and 15 trials included patients predominantly from Asia. The rest of the trials were reported to include patients from Europe (5 studies) and America (3 studies). The length of follow-up ranged from 12 to 54 weeks. Most of the trials included a comparison between two interventions. Only 5 trials mentioned comparisons among three interventions. All trials involved 10 drugs as follows: infliximab (INF), etanercept (ETN), adalimumab (ADA), golimumab (GOL), tocilizumab (TCZ), abatacept (ABT), certolizumab pegol (CZP), methotrexate (MTX), conventional disease-modifying anti-rheumatic drugs (CDMARDs) and placebo (PBO). The full network of comparisons categorized in different outcomes was shown in Figure 2.

\section{American College of Rheumatology $20 \%$ response rate (ACR20)}

ACR20 was normally defined as a $20 \%$ improvement for patients with rheumatoid arthritis. The estimated ORs with $95 \%$ Crls of ACR20 for each comparison are shown in the lower panel of Table II. Among these 15 therapies, $\mathrm{ABT}+\mathrm{MTX}(\mathrm{OR}=5.42,95 \% \mathrm{Crl}: 2.12-14.0), \mathrm{ADA}$ $(\mathrm{OR}=4.31,95 \% \mathrm{Crl}: 2.53-7.39), \mathrm{ADA}+\mathrm{MTX}$ $(\mathrm{OR}=5.81,95 \% \mathrm{Crl}: 2.39-14.7), \mathrm{CZP}(\mathrm{OR}=11.3$, 95\% Crl: 4.48-28.8), CZP + MTX (OR = 9.68, 95\% Crl: 3.86-24.5), ETN (OR $=4.22,95 \% \mathrm{Crl}$ : 2.23-8.17), ETN + MTX (OR = 6.31, 95\% Crl: 3.1014.0), GOL + MTX (OR = 6.23, 95\% Crl: 2.46-15.6), INF + MTX (OR = 5.75, 95\% Crl: 2.39-14.1), TCZ $(\mathrm{OR}=5.64,95 \% \mathrm{Crl}: 2.8-11.47)$ and $\mathrm{TCZ}+\mathrm{MTX}$ $(\mathrm{OR}=7.10,95 \% \mathrm{Crl}: 3.16-16.1)$ revealed superior efficacy under the endpoint of ACR20 compared with PBO. In addition, CZP + MTX was more efficacious than ETN when comparing ACR20 (OR = 2.29, 95\% Crl: 1.03-5.10).

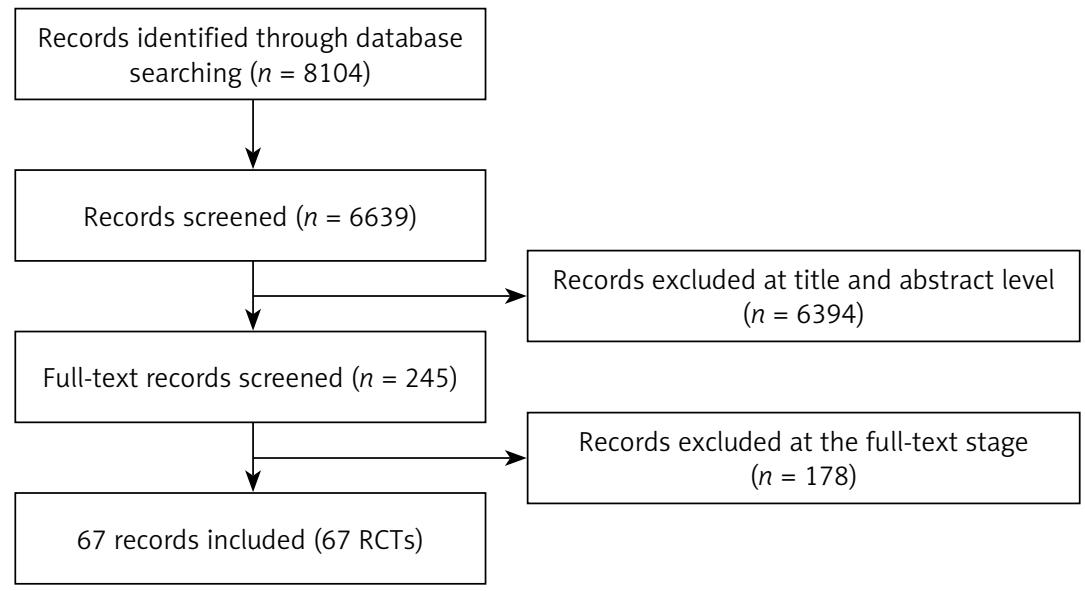

Figure 1. Flow diagram of study inclusion 


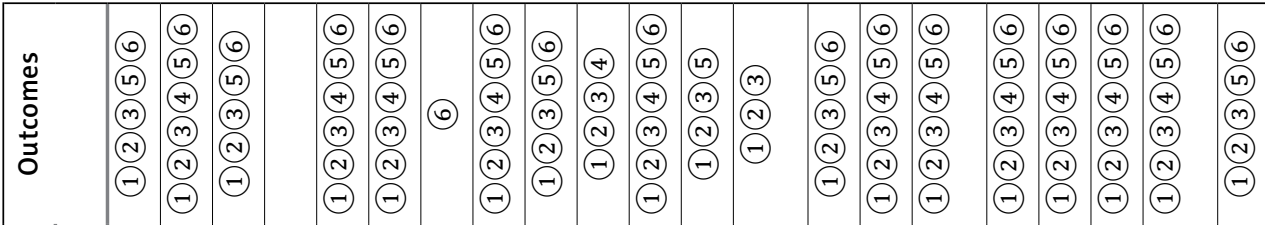

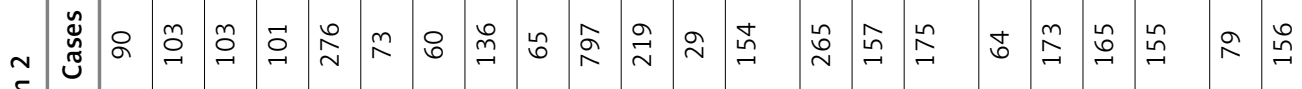

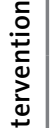

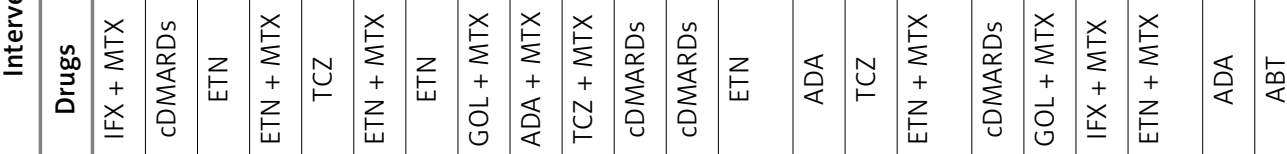

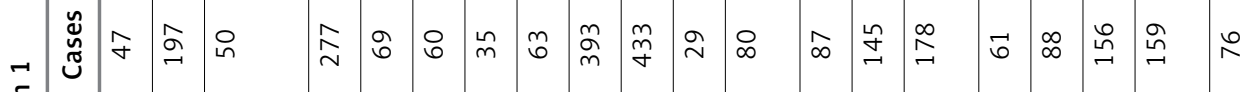

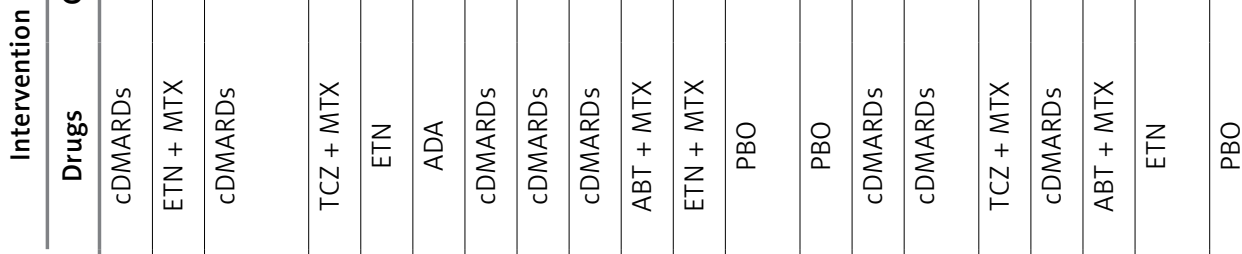

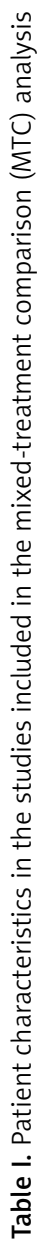

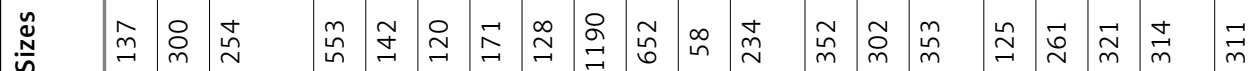

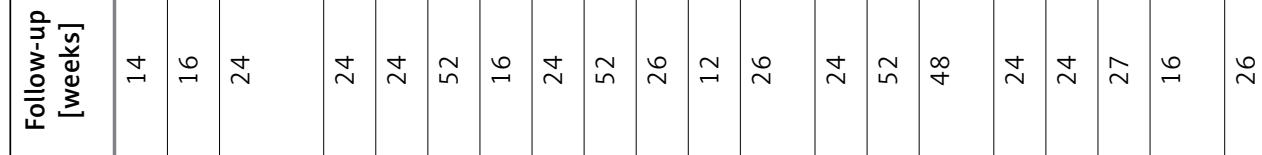

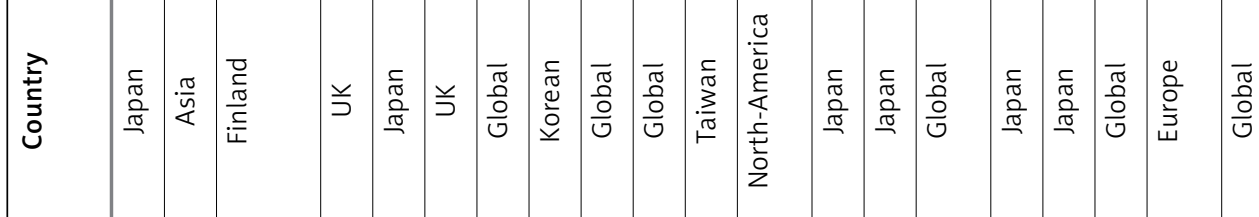

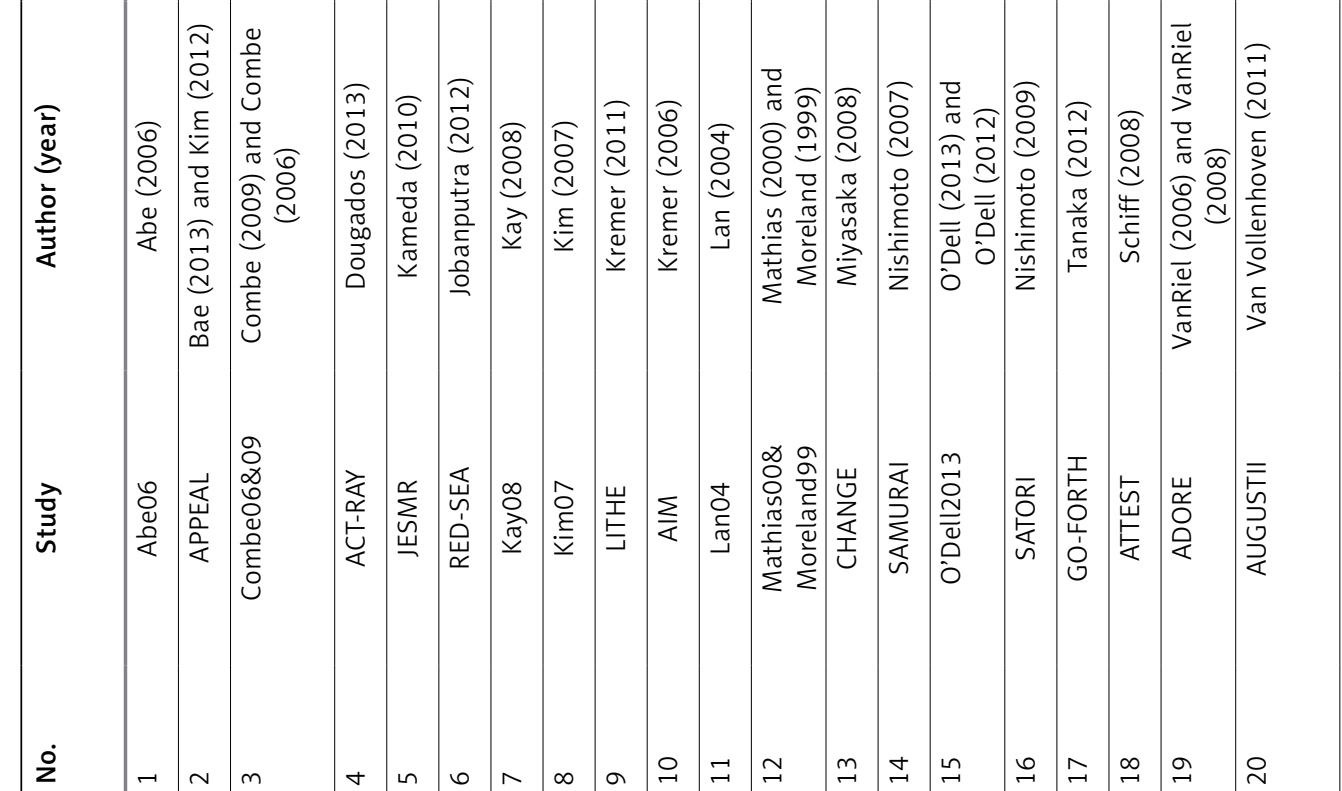




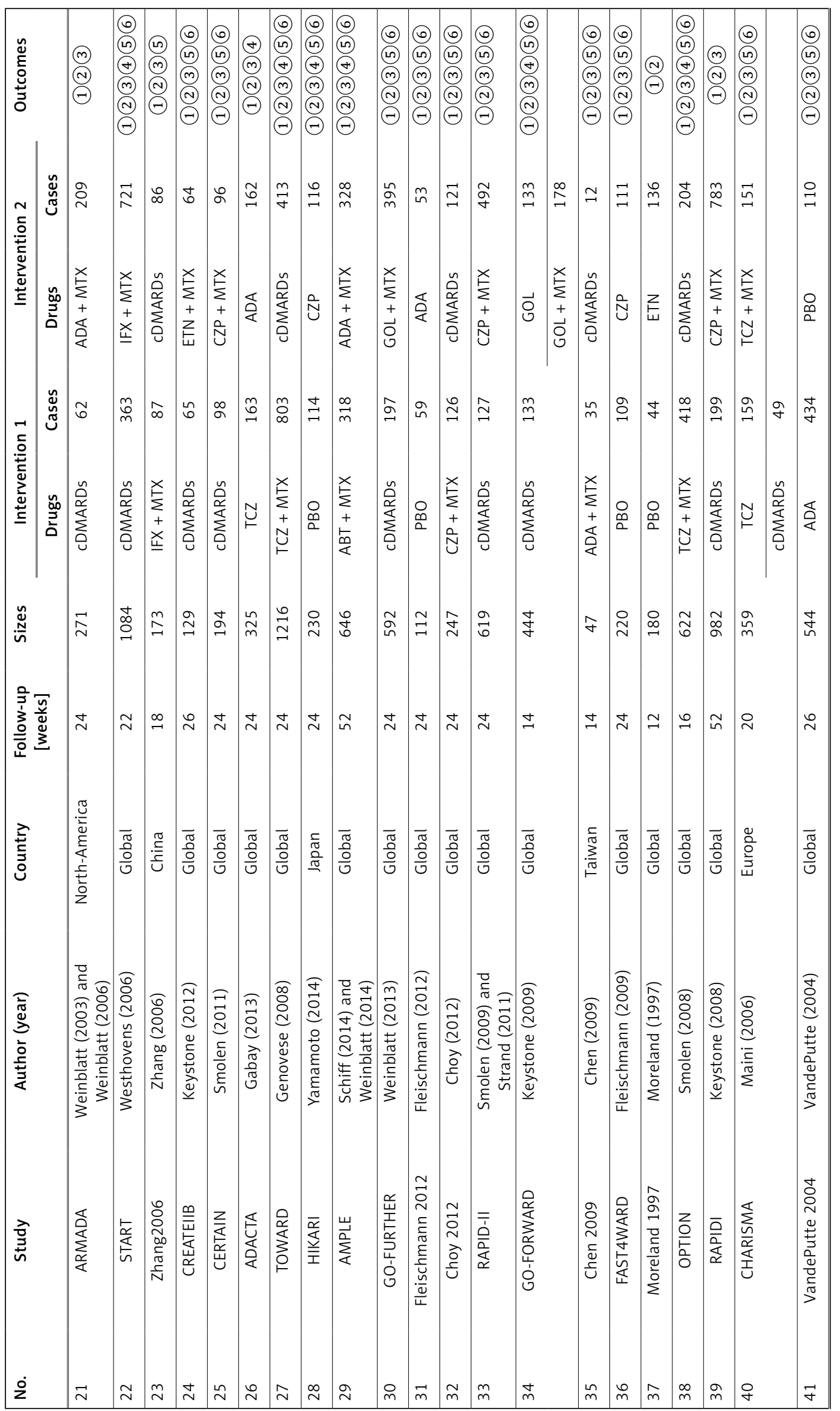




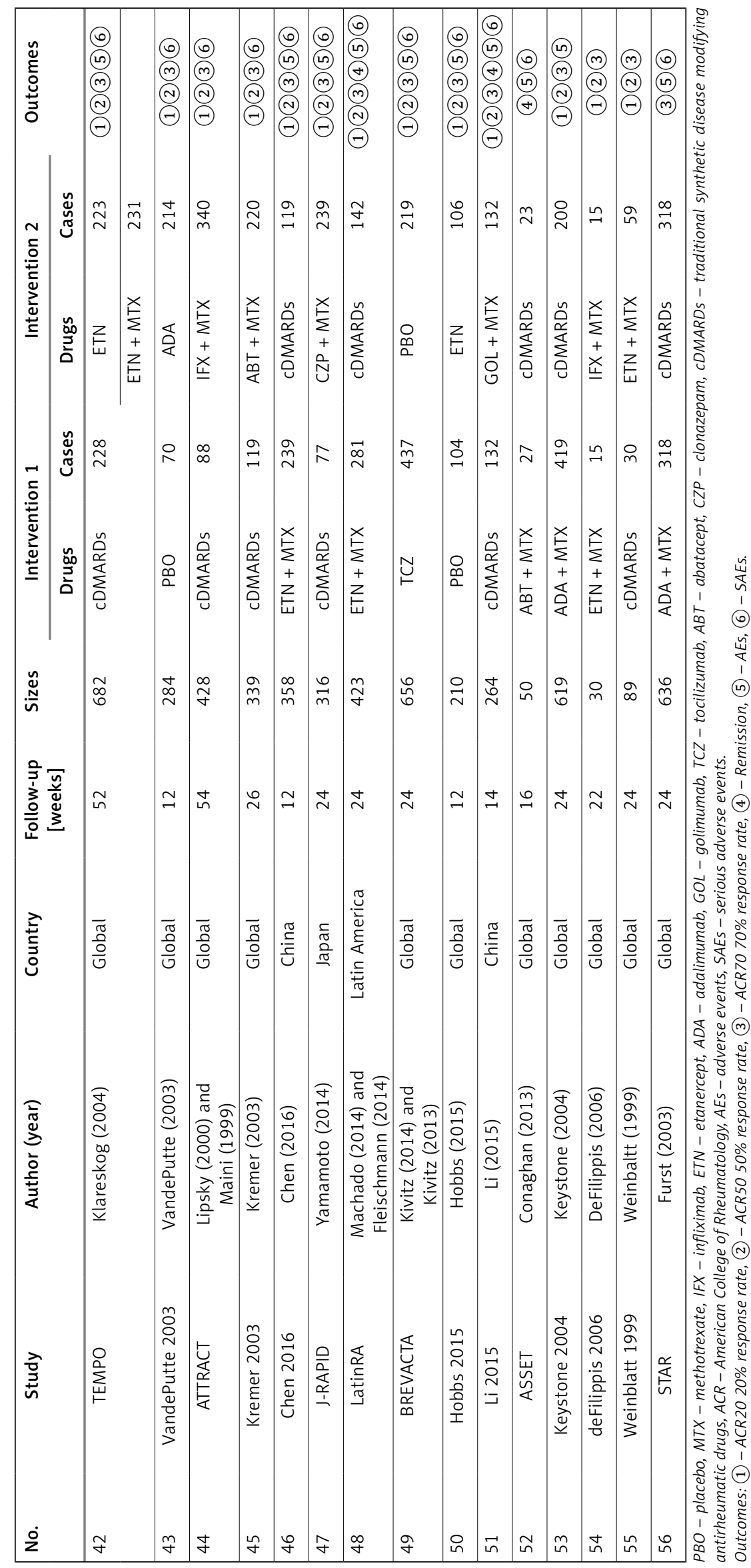




\section{American College of Rheumatology 50\% response rate (ACR50)}

Base on the upper panel of Table II, PBO showed the worst performance for ACR50 compared with all therapies except cDMARDs (OR $=1.86,95 \% \mathrm{Crl}$ : 0.94-3.71). As for cDMARDs, it revealed worse efficacy than the other treatments except ABT, GOL and PBO. In addition, ETN + MTX, CZP + MTX and TCZ + MTX were superior to ETN for the efficacy of ACR50 (ETN + MTX: OR = 1.59, 95\% Crl: 1.01-2.56; CZP + MTX: OR $=2.27,95 \%$ Crl: 1.07-4.76; TCZ + MTX: OR $=2.10,95 \%$ Crl: $1.06-4.01$.
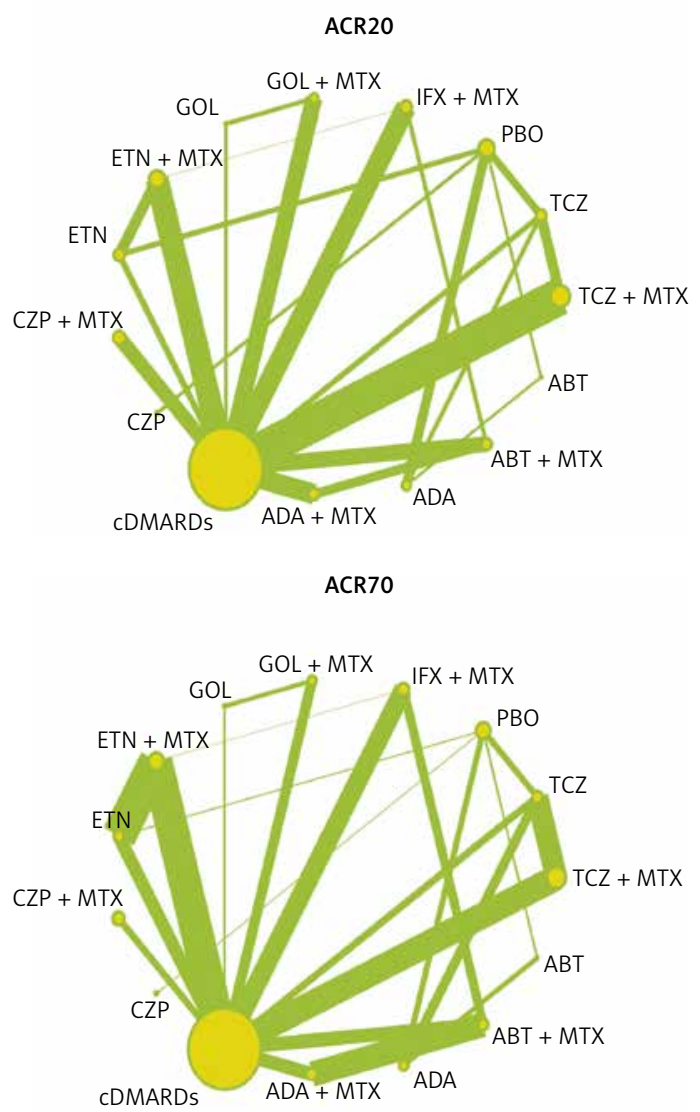

AEs

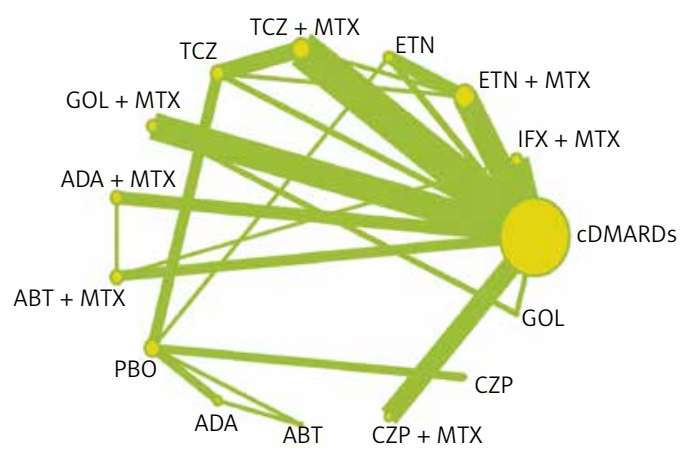

\section{American College of Rheumatology $70 \%$ response rate (ACR70)}

As shown in the lower panel of Table III, only CDMARDs (OR $=2.41,95 \%$ Crl: 0.91-7.10) demonstrated no statistically significant difference from PBO. Similarly, all the therapies appeared superior to CDMARDs when comparing ACR70, except for ABT $(\mathrm{OR}=1.60,95 \% \mathrm{Crl}: 0.32-7.54), \mathrm{ADA}(\mathrm{OR}=2.77$, $95 \% \mathrm{Crl}: 0.93-8.08)$ and GOL (OR $=2.69,95 \% \mathrm{Crl}$ : 0.37-10.3) Additionally, CZP enjoyed obvious superiority to $\mathrm{ABT}(\mathrm{OR}=0.05,95 \% \mathrm{Crl}: 0.01-0.62)$ and $\operatorname{ADA}(\mathrm{OR}=0.09,95 \% \mathrm{Crl}: 0.01-0.84)$.
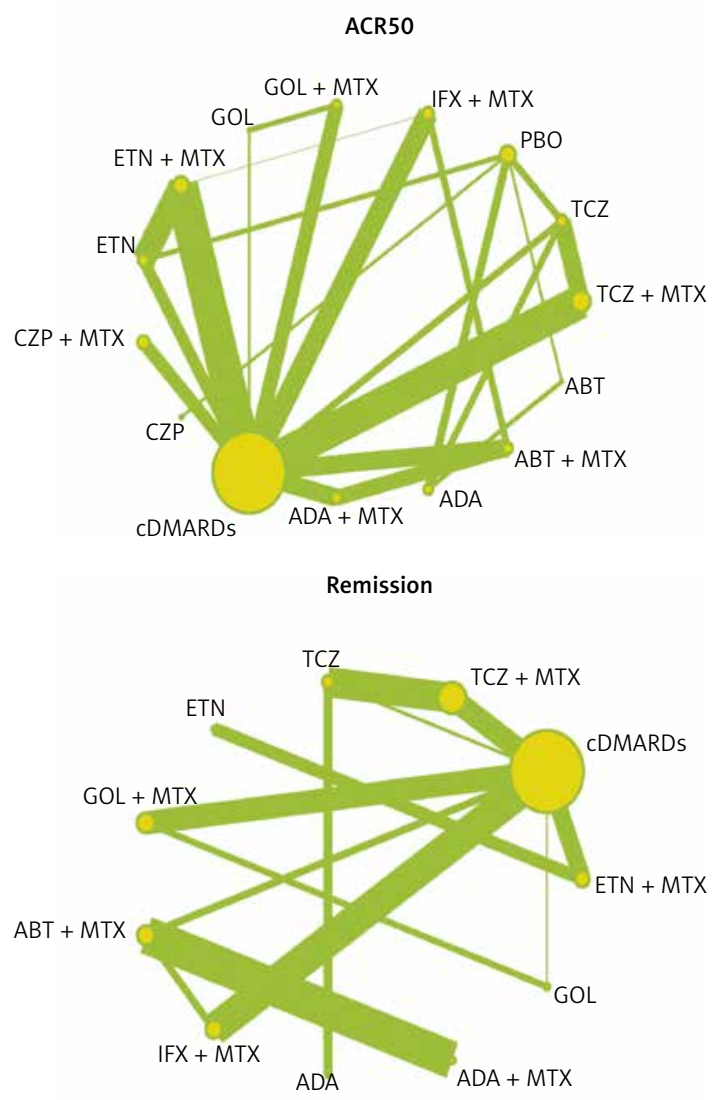

SAEs

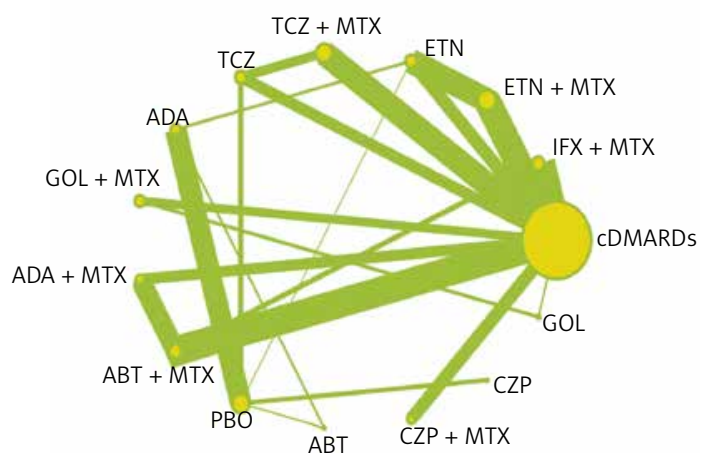

Figure 2. Full network of comparisons categorized in different outcomes. The width of the lines is proportional to the number of trials comparing each pair of treatments; the area of circles represents the cumulative number of patients for each intervention 


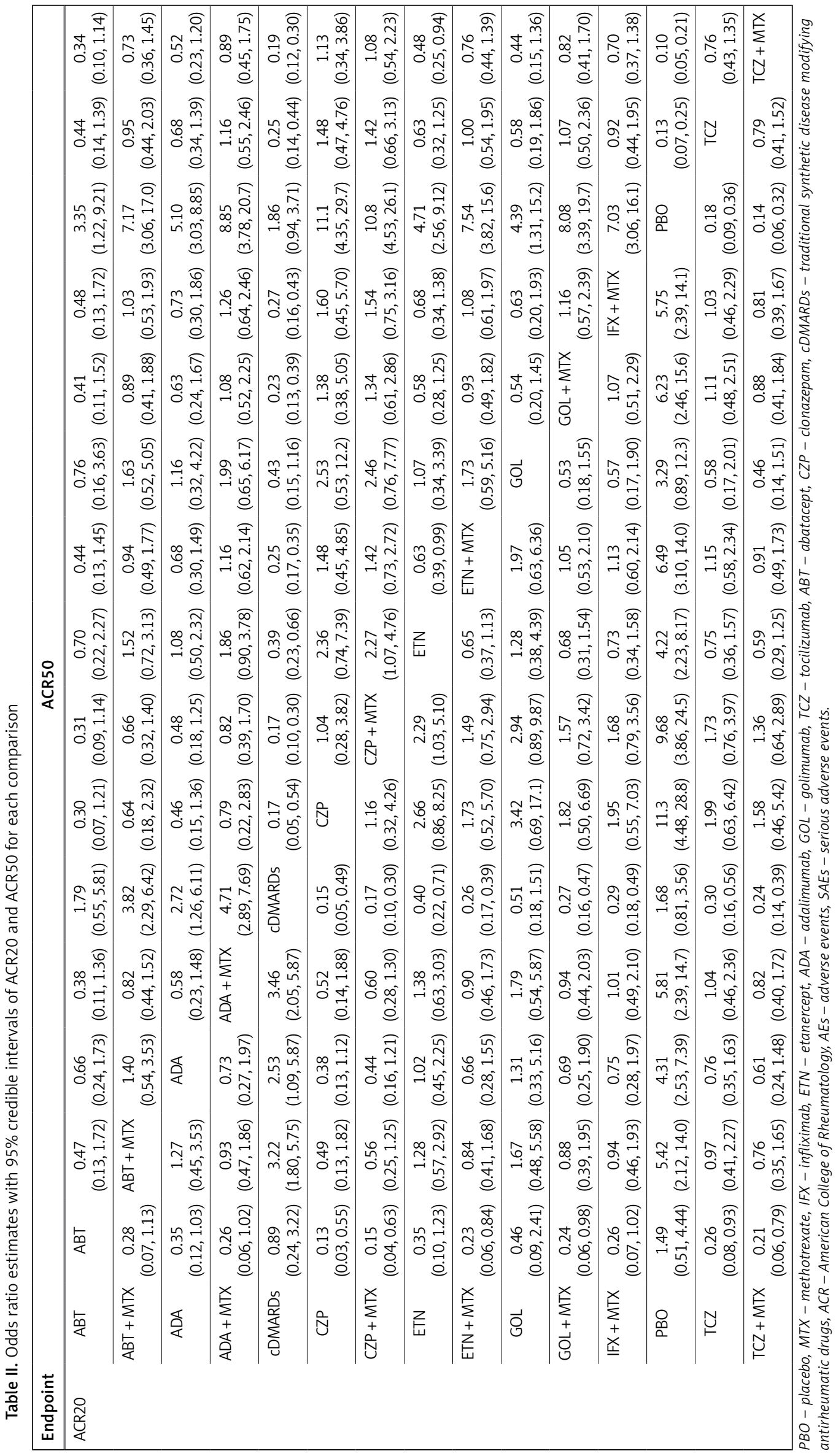


Efficacy and safety of various anti-rheumatic treatments for patients with rheumatoid arthritis: a network meta-analysis

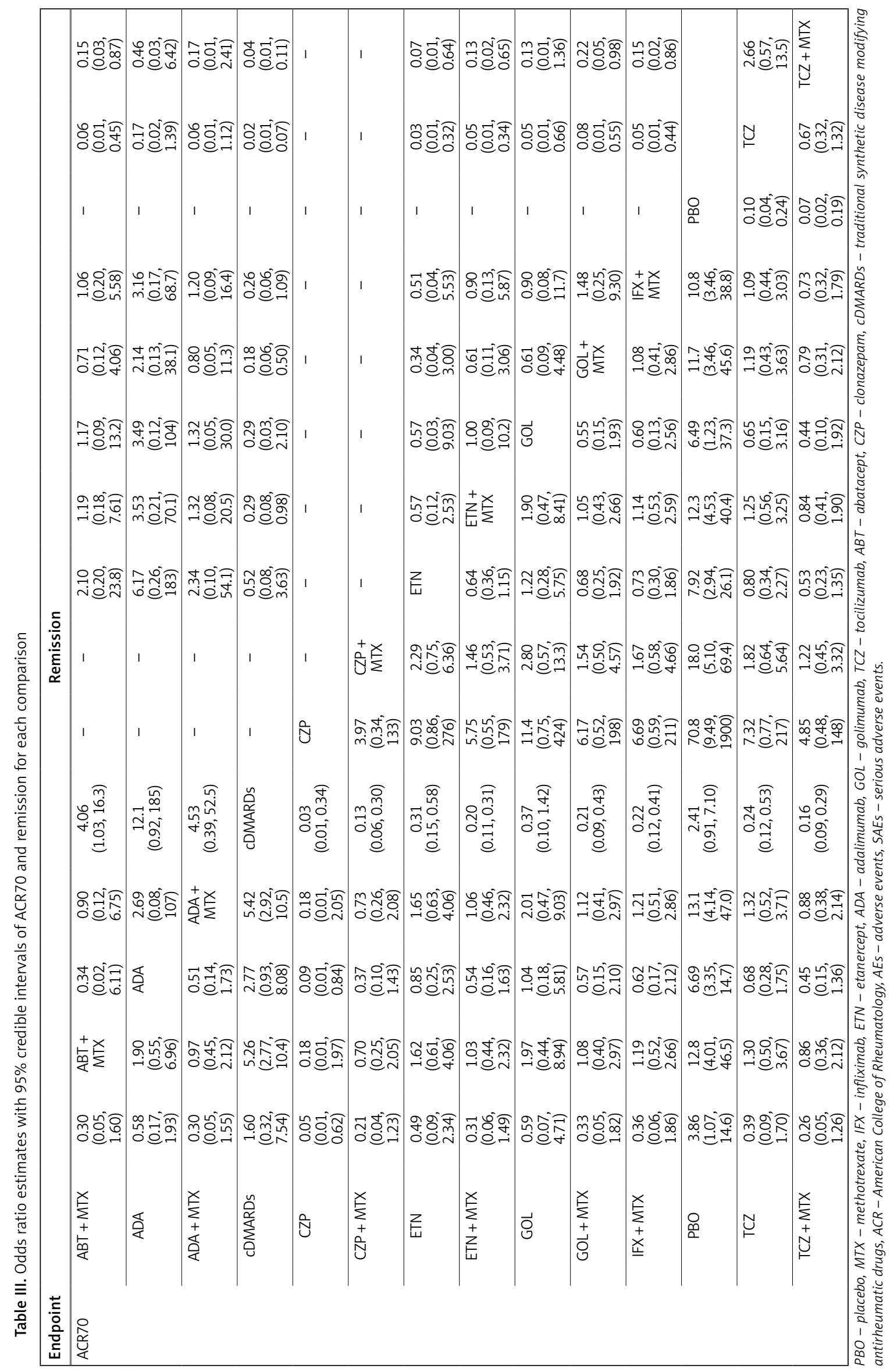




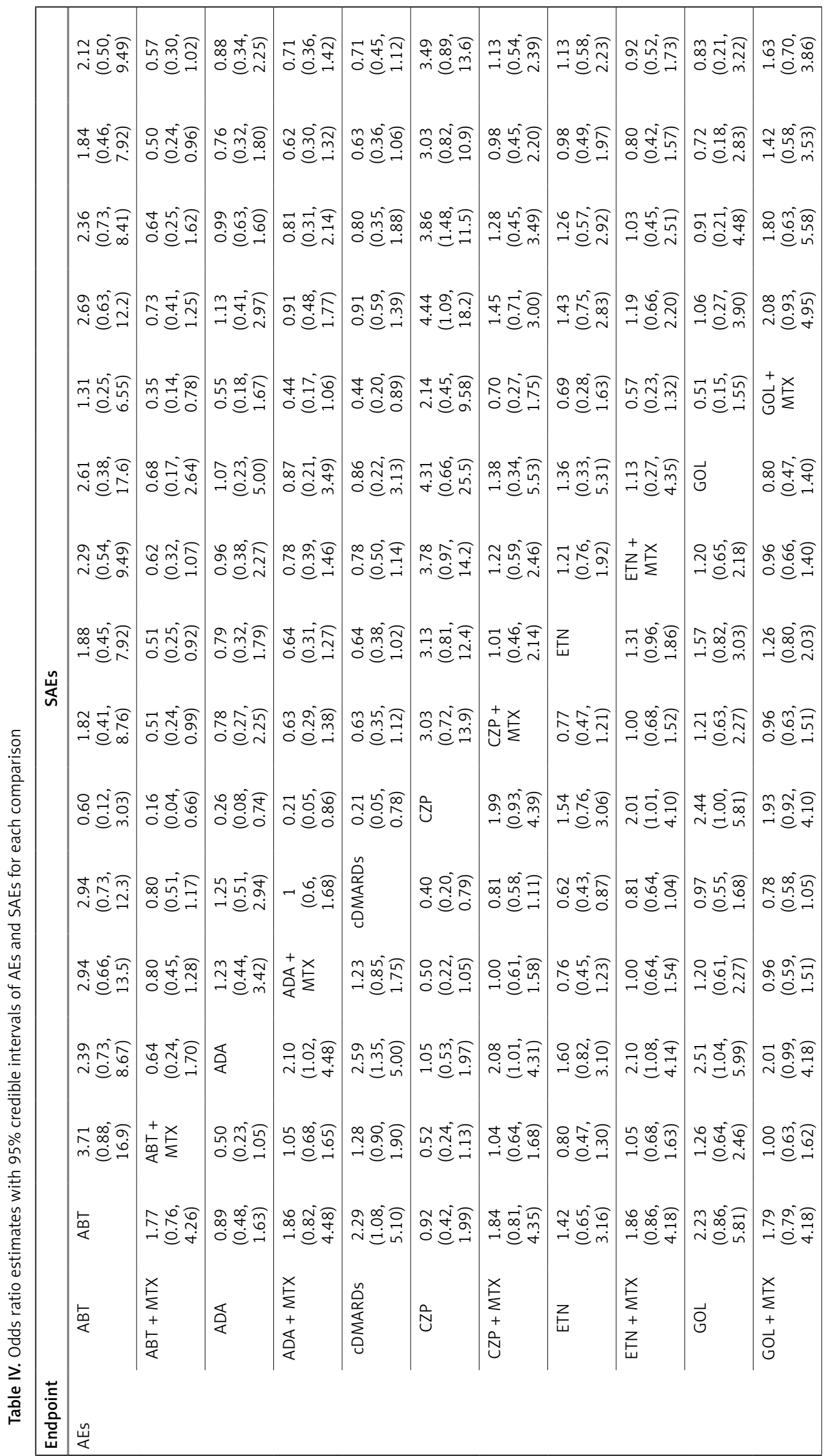




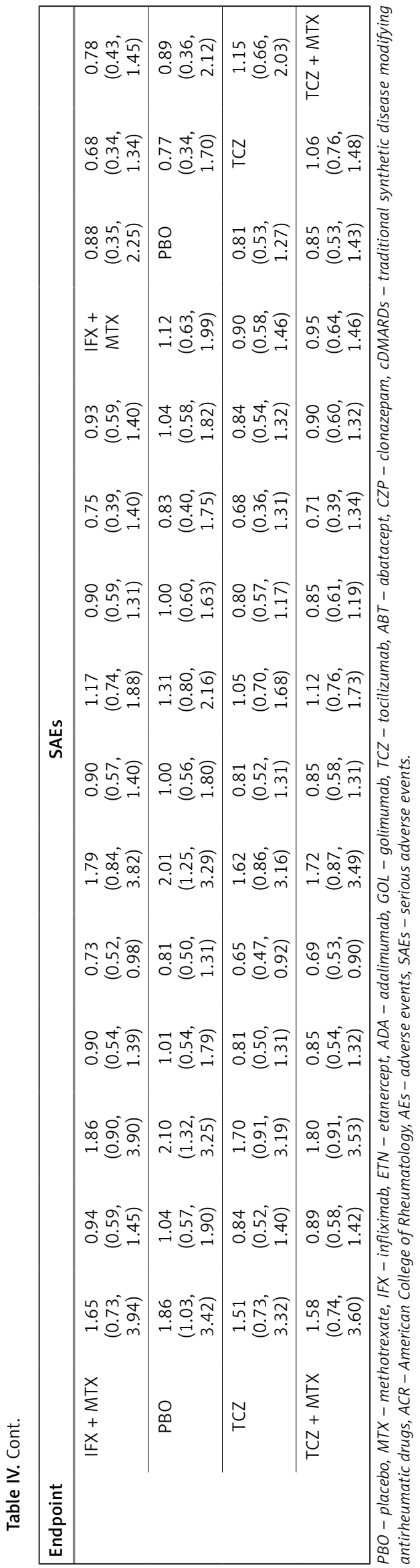

\section{Remission}

The efficacy endpoint of remission was evaluated among 11 treatments as displayed in the upper panel of Table III. It could be observed that TCZ and TCZ + MTX were significantly better than $\mathrm{ABT}+\mathrm{MTX}(\mathrm{OR}=0.06,95 \% \mathrm{Crl}: 0.01-0.45 ; \mathrm{OR}=$ 0.15, 95\% Crl: 0.03-0.87), cDMARDs (OR $=0.01$, 95\% Crl: 0.01-0.07; OR = 0.04, 95\% Crl: 0.01-0.11), ETN (OR $=0.03,95 \%$ Crl: 0.01-0.32; OR $=0.07$, 95\% Crl: 0.01-0.64), ETN + MTX (OR = 0.05, 95\% Crl: 0.01-0.34; OR = 0.13, 95\% Crl: 0.02-0.65), $\mathrm{GOL}+\mathrm{MTX}(\mathrm{OR}=0.08,95 \% \mathrm{Crl}: 0.01-0.55 ; \mathrm{OR}=$ $0.22,95 \% \mathrm{Crl}: 0.05-0.98)$ and IFX + MTX (OR $=0.05$, 95\% Crl: 0.01-0.44; OR = 0.15, 95\% Crl: 0.02-0.86) in disease remission. However, there was no particular evidence to confirm which one of TCZ and TCZ + MTX was better. ABT + MTX (OR = 0.25, 95\% Crl: 0.06-0.97), ETN + MTX (OR $=0.29,95 \%$ Crl: 0.08$0.98)$ and GOL + MTX (OR $=0.18,95 \%$ Crl: $0.06-$ $0.50)$ also presented greater remission of pain compared to CDMARDs. Additionally, GOL $(\mathrm{OR}=0.05$, 95\% Crl: 0.01-0.66) was less efficacious than TCZ.

\section{Adverse events (AEs)}

The safety outcomes are shown in Table IV. Statistically, ABT (OR = 1.86, 95\% Crl: 1.03-3.42), ADA (OR $=2.10,95 \% \mathrm{Crl}: 1.32-3.25)$ and CZP $(\mathrm{OR}=2.01,95 \% \mathrm{Crl}: 1.25-3.29)$ presented a higher risk of AEs than PBO. ADA was more likely to cause adverse events than ADA + MTX (OR = 2.10, 95\% Crl: 1.02-4.48), cDMARDs (OR $=2.59$, 95\% Crl: $1.35-5.00), C Z P+M T X(O R=2.08,95 \%$ $\mathrm{Crl}: 1.01-4.31), \mathrm{ETN}+\mathrm{MTX}(\mathrm{OR}=2.10,95 \% \mathrm{Crl}$ : 1.08-4.14), GOL (OR = 2.51, 95\% Crl: 1.04-5.99) and PBO (OR $=2.10,95 \% \mathrm{Crl}: 1.32-3.25)$. In comparison with ETN + MTX (OR $=2.01,95 \% \mathrm{Crl}$ : 1.01-4.10) and GOL (OR = 2.44, 95\% Crl: 1.005.81) more patients taking CZP dropped out due to AEs. Moreover, the safety of cDMARDs for adverse events was superior to CZP (OR $=0.40,95 \% \mathrm{Crl}$ : 0.20-0.79), ETN (OR = 0.62, 95\% Crl: 0.43-0.87), IFX + MTX (OR = 0.73, 95\% Crl: 0.52-0.98), TCZ $(\mathrm{OR}=0.65,95 \% \mathrm{Crl}: 0.47-0.92)$ and $\mathrm{TCZ}+\mathrm{MTX}$ $(\mathrm{OR}=0.69,95 \% \mathrm{Crl}: 0.53-0.90)$.

\section{Serious adverse events (SAEs)}

The comparison of SAEs for all the treatments is displayed in the upper panel of Table IV. CZP (OR $=3.86,95 \% \mathrm{Crl}: 1.48-11.5)$ presented a worse performance than PBO (OR $=0.26,95 \% \mathrm{Crl}$ : 0.090.68), INF + MTX (OR = 0.23, 95\% Crl: 0.06-0.91), CDMARDs (OR $=0.21,95 \% \mathrm{Crl}: 0.05-0.78), \mathrm{ADA}+$ $\operatorname{MTX}(\mathrm{OR}=0.21,95 \% \mathrm{Crl}: 0.05-0.86), \mathrm{ADA}(\mathrm{OR}=$ $0.26,95 \% \mathrm{Crl}: 0.08-0.74)$ and $A B T+M T X(O R=$ 0.16, 95\% Crl: 0.04-0.66). In contrast, ABT + MTX was more efficacious in reducing the SAEs in comparison with CZP + MTX (OR $=0.51,95 \% \mathrm{Crl}$ : $0.24-$ 
0.99), ETN (OR $=0.51,95 \% \mathrm{Crl}: 0.25-0.92), \mathrm{GOL}+$ $\operatorname{MTX}(\mathrm{OR}=0.35,95 \% \mathrm{Crl}: 0.14-0.78)$ and TCZ (OR = 0.50, 95\% Crl: 0.24-0.96). Furthermore, cDMARDs (OR $=0.44,95 \%$ Crl: 0.20-0.89) worked better than $\mathrm{GOL}+\mathrm{MTX}$ in withdrawal due to SAEs.

\section{Relative ranking analysis}

Relative ranking of the treatments is assessed by SUCRA in Table V. Since CZP + MTX not only had high efficacy in ACR20 (83.3\%), ACR50 (84.2\%) and ACR70 (75.1\%) but also performed well in AEs and SAEs, we recommend CZP + MTX as the optimal drug therapy. Another alternative was TCZ + MTX for the same reason. By contrast, ABT was regarded as the worst choice in treating RA because of its low probability in efficacy outcomes (ACR20: $10.8 \%, A C R 50=2.4 \%, A C R 70=20.0 \%)$ and safety outcomes (AEs $=14.8 \%$, SAEs $=17.2 \%$ ). Also, CDMARDs are not recommended due to their low efficacy, though their safety seemed to be superior.

\section{Consistency test}

The node-splitting method was used to evaluate the consistency level between direct and indirect evidence. $P$-values $<0.05$ implied the existence of a significant inconsistency. As listed in Table VI, a significant inconsistency did exist in the analysis of remission and AEs. As for remission, obvious inconsistency was found in the comparisons between TCZ and cDMARDs $(p=0.013)$, TCZ + MTX and CDMARDs $(p=0.015)$, as well as TCZ + MTX and TCZ $(p=0.019)$. On the other hand, no consistency between ETN + MTX and CDMARDs $(p<0.001)$, TCZ and ETN + MTX $(p=0.034), \mathrm{TCZ}+\mathrm{MTX}$ and ETN + MTX $(p=0.025)$ was demonstrated when comparing them with AEs. The results of the consistency test are also visually presented in Figure 3 with net heat plots, which indicated the same results as in Table VI.

\section{Publication bias}

The estimate of publication bias was performed by the symmetry characteristics of the dots representing included trials with different colors in the funnel plots. According to Figure 4, all of the funnel plots were focused in the triangle funnel areas in left and right directions, which suggested that the distribution of dots verified no significant publication bias or small study effect among the trials in ACR20, ACR50, ACR70, AEs, SAEs and remission.

\section{Evaluation of the methodological quality of eligible studies}

The Jadad scale was used to appraise the methodological quality of included studies, and the scores of the Jadad scale of each individual study

Table V. Relative ranking of the treatments assessed by surface under cumulative ranking curve area

\begin{tabular}{|c|c|c|c|c|c|c|}
\hline Treatments & ACR20 & ACR50 & ACR70 & Remission & AEs & SAEs \\
\hline ABT & 0.108 & 0.239 & 0.200 & - & 0.148 & 0.172 \\
\hline$A B T+M T X$ & 0.510 & 0.564 & 0.605 & 0.402 & 0.549 & 0.852 \\
\hline ADA & 0.392 & 0.375 & 0.326 & 0.617 & 0.077 & 0.551 \\
\hline$A D A+M T X$ & 0.554 & 0.722 & 0.620 & 0.428 & 0.606 & 0.693 \\
\hline cDMARDs & 0.108 & 0.084 & 0.089 & 0.050 & 0.862 & 0.727 \\
\hline CZP & 0.818 & 0.796 & 0.882 & - & 0.099 & 0.051 \\
\hline$C Z P+M T X$ & 0.833 & 0.842 & 0.751 & - & 0.602 & 0.365 \\
\hline ETN & 0.359 & 0.307 & 0.358 & 0.220 & 0.295 & 0.356 \\
\hline $\mathrm{ETN}+\mathrm{MTX}$ & 0.634 & 0.611 & 0.597 & 0.364 & 0.556 & 0.506 \\
\hline $\mathrm{GOL}$ & 0.303 & 0.332 & 0.332 & 0.360 & 0.747 & 0.567 \\
\hline $\mathrm{GOL}+\mathrm{MTX}$ & 0.594 & 0.660 & 0.561 & 0.501 & 0.555 & 0.186 \\
\hline IFX + MTX & 0.547 & 0.546 & 0.511 & 0.385 & 0.466 & 0.637 \\
\hline PBO & 0.023 & 0.004 & 0.005 & - & 0.613 & 0.545 \\
\hline $\mathrm{TCZ}$ & 0.532 & 0.609 & 0.470 & 0.890 & 0.352 & 0.346 \\
\hline $\mathrm{TCZ}+\mathrm{MTX}$ & 0.685 & 0.809 & 0.693 & 0.784 & 0.407 & 0.445 \\
\hline
\end{tabular}

PBO - placebo, MTX - methotrexate, IFX - infliximab, ETN - etanercept, ADA - adalimumab, GOL - golimumab, TCZ - tocilizumab, $A B T$ - abatacept, CZP - clonazepam, CDMARDs - traditional synthetic disease modifying antirheumatic drugs, ACR - American College of Rheumatology, AEs - adverse events, SAEs - serious adverse events. 
ACR20

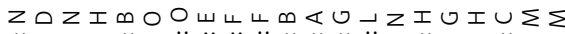

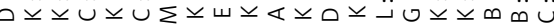

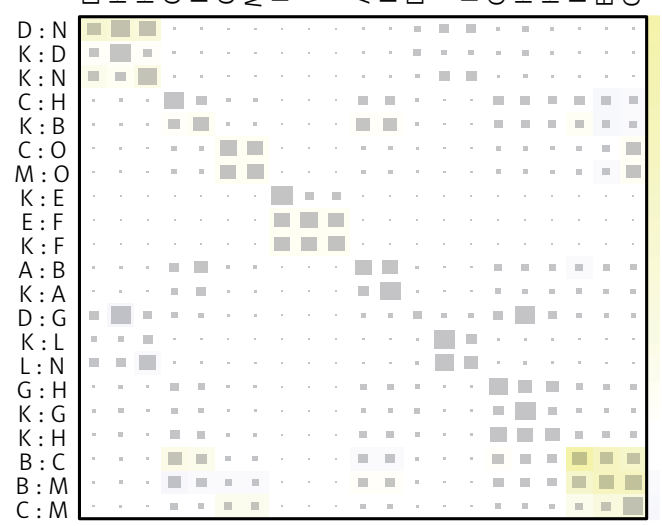

ACR70

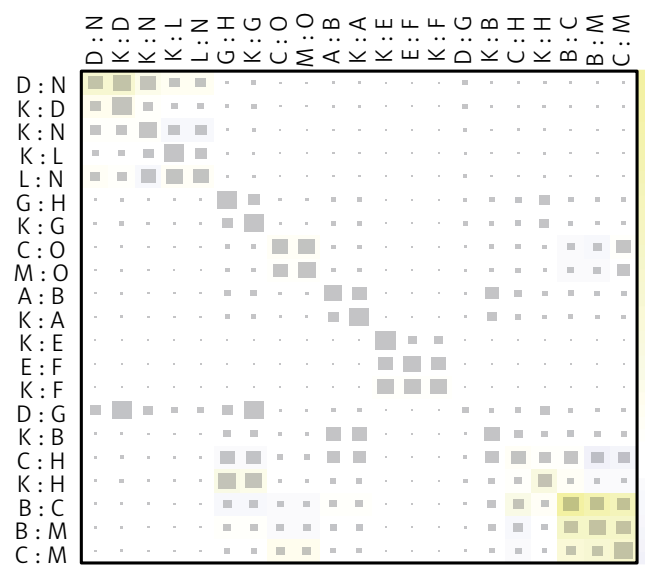

AEs

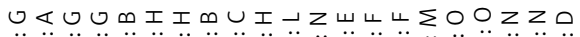

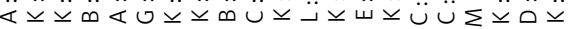

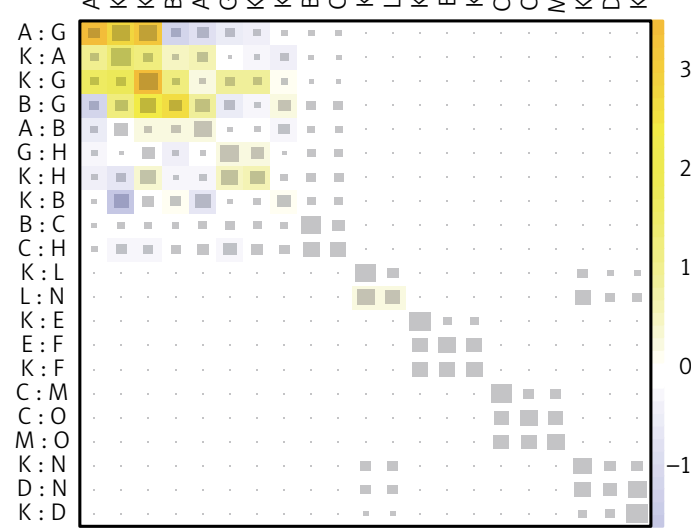

ACR50

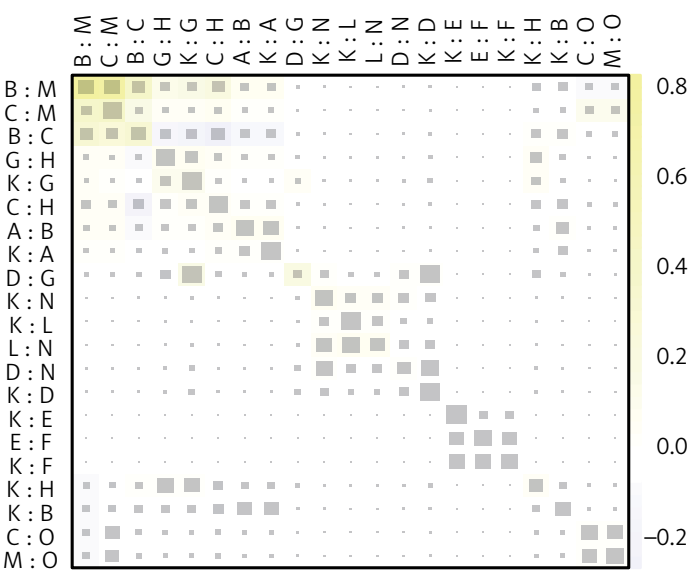

Remission

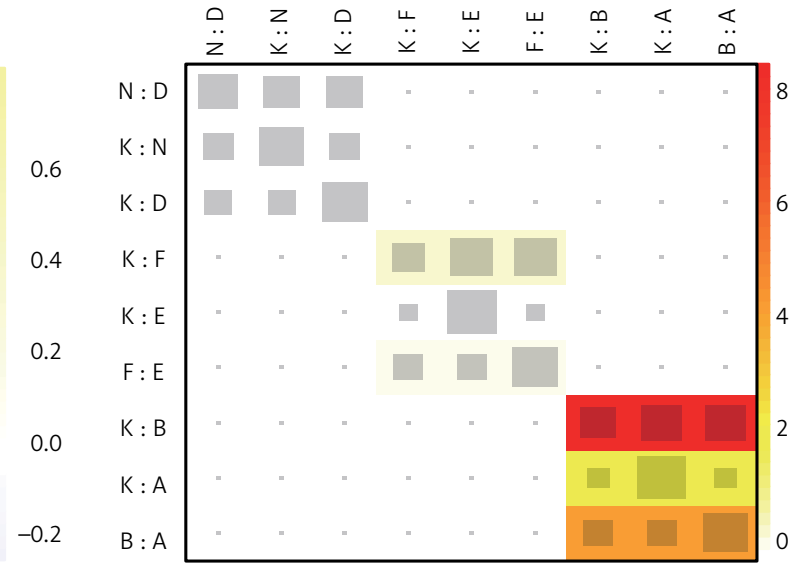

SAEs

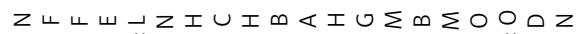

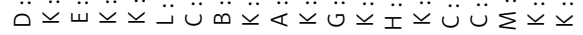

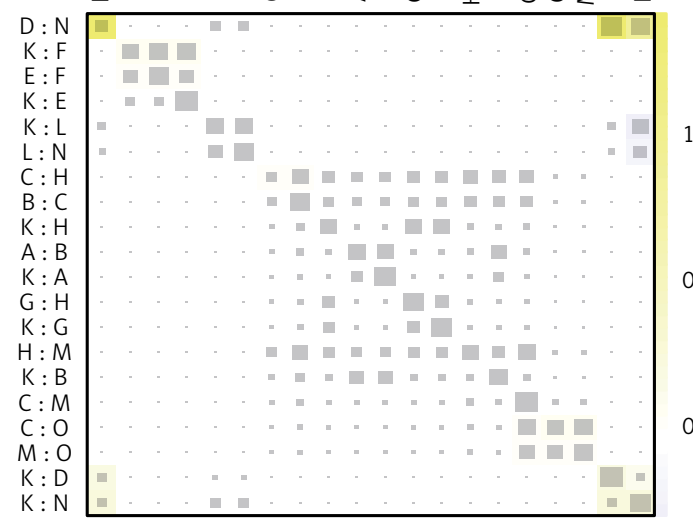

Figure 3. Results of consistency analysis by heat plot. Inconsistency between direct and indirect evidence was assessed using the net heat plots, which visually displayed the inconsistency level with different colors. The more vibrant the color was, the more serious was the indicated inconsistency

$A-T C Z+M T X, B-T C Z, C-P B O, D-I F X+M T X, E-G O L+M T X, F-G O L, G-E T N+M T X, H-E T N, I-C Z P+M T X, J-C Z P$, $K-C D M A R D S, L-A D A+M T X, M-A D A, N-A B T+M T X, O-A B T$ 
Kexun Ma, Ling Li, Chunhui Liu, Lingling Zhou, Xueping Zhou

Table VI. Results of consistency analysis by node-splitting plot

\begin{tabular}{|c|c|c|c|c|c|}
\hline Endpoint & Comparison & Direct OR $(95 \% \mathrm{Cl})$ & $\begin{array}{c}\text { Indirect OR } \\
(95 \% \mathrm{Crl})\end{array}$ & $\begin{array}{c}\text { Network OR } \\
(95 \% \mathrm{Crl})\end{array}$ & $P$-value \\
\hline \multirow[t]{16}{*}{ ACR20 } & $A D A+M T X$ vs. ABT $+M T X$ & $1.10(0.35,3.50)$ & $1.10(0.48,2.70)$ & $1.10(0.53,2.10)$ & 0.899 \\
\hline & cDMARDs vs. ABT + MTX & $0.34(0.17,0.68)$ & $0.29(0.07,1.10)$ & $0.31(0.17,0.56)$ & 0.819 \\
\hline & cDMARDs vs. ADA + MTX & $0.29(0.16,0.52)$ & $0.31(0.08,1.10)$ & $0.29(0.17,0.49)$ & 0.928 \\
\hline & ETN vs. cDMARDs & $2.50(1.00,6.10)$ & $2.40(1.00,5.90)$ & $2.50(1.40,4.60)$ & 0.963 \\
\hline & ETN + MTX vs. cDMARDs & $3.80(2.50,5.70)$ & $4.70(1.30,15.0)$ & $3.80(2.60,5.70)$ & 0.723 \\
\hline & ETN + MTX vs. ETN & $1.50(0.86,2.90)$ & $1.20(0.27,4.90)$ & $1.50(0.89,2.70)$ & 0.751 \\
\hline & IFX + MTX vs. ABT + MTX & $0.72(0.22,2.40)$ & $1.30(0.53,3.20)$ & $1.10(0.51,2.30)$ & 0.425 \\
\hline & IFX + MTX vs. cDMARDs & $3.50(2.00,5.80)$ & $3.90(0.52,26.0)$ & $3.50(2.00,5.60)$ & 0.933 \\
\hline & IFX + MTX vs. ETN + MTX & $1.00(0.15,7.30)$ & $0.88(0.46,1.70)$ & $0.90(0.47,1.70)$ & 0.871 \\
\hline & PBO vs. ADA & $0.22(0.12,0.38)$ & $0.41(0.10,1.70)$ & $0.23(0.13,0.40)$ & 0.423 \\
\hline & PBO vs. ETN & $0.22(0.10,0.48)$ & $0.29(0.08,1.10)$ & $0.23(0.13,0.45)$ & 0.691 \\
\hline & TCZ vs. ADA & $1.90(0.53,6.20)$ & $1.00(0.34,2.70)$ & $1.30(0.62,2.90)$ & 0.413 \\
\hline & TCZ vs. cDMARDs & $3.40(1.40,8.50)$ & $3.30(1.30,8.40)$ & $3.30(1.80,6.40)$ & 0.950 \\
\hline & TCZ vs. PBO & $3.50(1.10,11.0)$ & $7.70(3.10,18.0)$ & $5.60(2.80,12.0)$ & 0.274 \\
\hline & TCZ + MTX vs. cDMARDs & $4.00(2.40,7.00)$ & $4.80(1.10,21.0)$ & $4.20(2.50,6.90)$ & 0.818 \\
\hline & TCZ + MTX vs. TCZ & $1.40(0.60,3.20)$ & $0.94(0.35,2.90)$ & $1.30(0.65,2.50)$ & 0.574 \\
\hline \multirow[t]{16}{*}{ ACR50 } & $A D A+M T X v s . A B T+M T X$ & $1.10(0.42,2.80)$ & $1.40(0.59,3.00)$ & $1.20(0.63,2.30)$ & 0.741 \\
\hline & cDMARDs vs. ABT + MTX & $0.29(0.16,0.56)$ & $0.22(0.07,0.74)$ & $0.26(0.15,0.45)$ & 0.679 \\
\hline & cDMARDs vs. ADA + MTX & $0.21(0.12,0.35)$ & $0.24(0.08,0.82)$ & $0.21(0.13,0.36)$ & 0.789 \\
\hline & ETN vs. cDMARDs & $2.20(0.96,4.80)$ & $3.00(1.40,6.80)$ & $2.50(1.50,4.50)$ & 0.565 \\
\hline & ETN + MTX vs. cDMARDs & $4.30(2.90,6.50)$ & $2.90(0.92,9.20)$ & $4.10(2.80,6.20)$ & 0.520 \\
\hline & ETN + MTX vs. ETN & $1.50(0.89,2.60)$ & $1.80(0.45,7.00)$ & $1.60(0.97,2.60)$ & 0.834 \\
\hline & IFX + MTX vs. ABT + MTX & $0.88(0.30,2.50)$ & $1.10(0.45,2.40)$ & $0.98(0.51,1.90)$ & 0.776 \\
\hline & IFX + MTX vs. cDMARDs & $3.60(2.10,6.00)$ & $5.80(0.84,41.0)$ & $3.80(2.40,6.00)$ & 0.630 \\
\hline & IFX + MTX vs. ETN + MTX & $1.40(0.24,9.40)$ & $0.87(0.47,1.60)$ & $0.93(0.52,1.60)$ & 0.606 \\
\hline & PBO vs. ADA & $0.17(0.09,0.30)$ & $0.41(0.10,1.40)$ & $0.19(0.11,0.32)$ & 0.223 \\
\hline & PBO vs. ETN & $0.21(0.10,0.47)$ & $0.20(0.06,0.64)$ & $0.21(0.10,0.40)$ & 0.920 \\
\hline & TCZ vs. ADA & $2.40(0.83,6.90)$ & $0.98(0.37,2.60)$ & $1.50(0.71,3.00)$ & 0.199 \\
\hline & TCZ vs. cDMARDs & $3.50(1.50,8.30)$ & $4.40(1.90,9.80)$ & $4.10(2.30,7.40)$ & 0.710 \\
\hline & TCZ vs. PBO & $5.00(1.90,14.0)$ & $9.80(4.40,22.0)$ & $7.70(4.00,15.0)$ & 0.299 \\
\hline & TCZ + MTX vs. CDMARDs & $4.60(3.00,7.20)$ & $9.10(2.70,33.0)$ & $5.30(3.40,8.30)$ & 0.299 \\
\hline & $\mathrm{TCZ}+\mathrm{MTX}$ vs. TCZ & $1.60(0.84,2.90)$ & $0.73(0.29,1.90)$ & $1.30(0.71,2.30)$ & 0.166 \\
\hline \multirow[t]{10}{*}{ ACR70 } & $A D A+M T X$ vs. ABT + MTX & $0.95(0.27,2.90)$ & $1.10(0.39,3.20)$ & $1.00(0.46,2.20)$ & 0.800 \\
\hline & cDMARDs vs. ABT + MTX & $0.19(0.08,0.42)$ & $0.16(0.04,0.69)$ & $0.19(0.09,0.37)$ & 0.809 \\
\hline & cDMARDs vs. ADA + MTX & $0.18(0.08,0.36)$ & $0.22(0.05,0.92)$ & $0.18(0.09,0.35)$ & 0.755 \\
\hline & ETN vs. CDMARDs & $2.60(1.00,8.00)$ & $4.40(1.70,13.0)$ & $3.30(1.70,6.60)$ & 0.463 \\
\hline & ETN + MTX vs. cDMARDs & $5.20(3.20,9.50)$ & $6.80(1.10,46.0)$ & $5.00(3.20,8.90)$ & 0.770 \\
\hline & ETN + MTX vs. ETN & $1.60(0.83,2.80)$ & $0.79(0.07,6.90)$ & $1.60(0.88,2.70)$ & 0.548 \\
\hline & $I F X+M T X$ vs. ABT + MTX & $1.20(0.32,4.30)$ & $0.58(0.21,1.60)$ & $0.85(0.38,1.90)$ & 0.355 \\
\hline & IFX + MTX vs. cDMARDs & $3.90(2.10,7.70)$ & $5.30(0.13,230)$ & $4.50(2.40,8.70)$ & 0.856 \\
\hline & IFX + MTX vs. ETN + MTX & $1.00(0.02,33.0)$ & $0.86(0.38,2.00)$ & $0.89(0.40,1.80)$ & 0.901 \\
\hline & PBO vs. ADA & $0.12(0.05,0.27)$ & $0.31(0.07,1.40)$ & $0.15(0.08,0.29)$ & 0.238 \\
\hline
\end{tabular}


Table VI. Cont.

\begin{tabular}{|c|c|c|c|c|c|}
\hline Endpoint & Comparison & Direct OR $(95 \% \mathrm{Cl})$ & $\begin{array}{c}\text { Indirect OR } \\
(95 \% \mathrm{Crl})\end{array}$ & $\begin{array}{c}\text { Network OR } \\
(95 \% \mathrm{Crl})\end{array}$ & $P$-value \\
\hline & PBO vs. ETN & $0.08(0.01,0.38)$ & $0.17(0.04,0.64)$ & $0.13(0.04,0.34)$ & 0.478 \\
\hline & TCZ vs. ADA & $2.20(0.70,7.10)$ & $0.85(0.22,3.60)$ & $1.50(0.58,3.60)$ & 0.299 \\
\hline & TCZ vs. cDMARDs & $3.20(1.10,8.80)$ & $5.40(1.50,18.0)$ & $4.10(1.90,8.40)$ & 0.494 \\
\hline & TCZ vs. PBO & $4.80(1.50,15.0)$ & $18.0(6.30,59.0)$ & $10.0(4.10,25.0)$ & 0.085 \\
\hline & TCZ + MTX vs. cDMARDs & $5.60(3.30,9.60)$ & $7.70(1.70,30.0)$ & $6.10(3.50,11.0)$ & 0.661 \\
\hline & $\mathrm{TCZ}+\mathrm{MTX}$ vs. TCZ & $1.60(0.83,3.50)$ & $0.90(0.30,3.00)$ & $1.50(0.73,3.20)$ & 0.396 \\
\hline \multirow[t]{4}{*}{ Remission } & IFX + MTX vs. ABT + MTX & $1.10(0.10,11.0)$ & $0.66(0.04,13.0)$ & $0.94(0.16,5.00)$ & 0.749 \\
\hline & TCZ vs. cDMARDs & $360(73.0,1800)$ & $12.0(2.60,71.0)$ & $70.0(13.0,380)$ & 0.013 \\
\hline & TCZ + MTX vs. CDMARDs & $15.0(7.10,37.0)$ & $430(47.0,3500)$ & $26.0(9.10,78.0)$ & 0.015 \\
\hline & TCZ + MTX vs. TCZ & $1.30(0.34,4.60)$ & $0.04(0.01,0.29)$ & $0.38(0.07,1.70)$ & 0.019 \\
\hline \multirow[t]{14}{*}{ AEs } & $A D A+M T X$ vs. ABT + MTX & $0.84(0.41,1.60)$ & $1.00(0.57,1.80)$ & $0.95(0.60,1.50)$ & 0.628 \\
\hline & cDMARDs vs. ABT + MTX & $0.83(0.53,1.30)$ & $0.65(0.30,1.50)$ & $0.77(0.53,1.10)$ & 0.611 \\
\hline & cDMARDs vs. ADA + MTX & $0.78(0.50,1.20)$ & $0.96(0.42,2.20)$ & $0.81(0.57,1.20)$ & 0.635 \\
\hline & ETN vs. cDMARDs & $2.00(1.20,3.50)$ & $1.30(0.85,2.20)$ & $1.60(1.20,2.40)$ & 0.236 \\
\hline & ETN + MTX vs. cDMARDs & $1.00(0.79,1.30)$ & $2.70(1.70,4.30)$ & $1.20(0.97,1.60)$ & $<0.001$ \\
\hline & ETN + MTX vs. ETN & $0.86(0.55,1.20)$ & $0.47(0.24,0.93)$ & $0.77(0.53,1.10)$ & 0.154 \\
\hline & IFX + MTX vs. ABT + MTX & $1.20(0.58,2.30)$ & $0.99(0.56,1.80)$ & $1.10(0.67,1.70)$ & 0.679 \\
\hline & PBO vs. ETN & $0.76(0.37,1.50)$ & $0.76(0.35,1.50)$ & $0.77(0.45,1.20)$ & 0.996 \\
\hline & TCZ vs. cDMARDs & $1.60(0.92,2.70)$ & $1.40(0.83,2.20)$ & $1.50(1.10,2.10)$ & 0.708 \\
\hline & TCZ vs. ETN + MTX & $0.68(0.36,1.30)$ & $1.50(0.97,2.40)$ & $1.20(0.85,1.80)$ & 0.034 \\
\hline & TCZ vs. PBO & $1.20(0.75,2.20)$ & $1.20(0.51,2.80)$ & $1.20(0.79,2.00)$ & 0.996 \\
\hline & TCZ + MTX vs. CDMARDs & $1.60(1.20,2.10)$ & $0.94(0.54,1.60)$ & $1.40(1.10,1.80)$ & 0.093 \\
\hline & TCZ + MTX vs. ETN + MTX & $0.61(0.30,1.10)$ & $1.40(0.99,2.00)$ & $1.20(0.84,1.60)$ & 0.025 \\
\hline & TCZ + MTX vs. TCZ & $0.86(0.58,1.30)$ & $1.00(0.53,1.90)$ & $0.95(0.68,1.30)$ & 0.619 \\
\hline \multirow[t]{14}{*}{ SAEs } & $A D A+M T X$ vs. ABT + MTX & $1.20(0.58,2.40)$ & $1.30(0.61,3.70)$ & $1.20(0.77,2.20)$ & 0.919 \\
\hline & cDMARDs vs. ABT + MTX & $1.30(0.80,2.30)$ & $1.20(0.42,3.00)$ & $1.20(0.83,1.90)$ & 0.885 \\
\hline & cDMARDs vs. ADA + MTX & $0.97(0.45,1.90)$ & $1.00(0.45,2.60)$ & $0.99(0.59,1.70)$ & 0.936 \\
\hline & ETN vs. ADA & $1.30(0.32,4.30)$ & $1.40(0.43,4.70)$ & $1.30(0.53,3.40)$ & 0.904 \\
\hline & ETN vs. cDMARDs & $1.70(0.92,3.60)$ & $1.40(0.55,4.00)$ & $1.60(0.97,2.7)$ & 0.760 \\
\hline & ETN + MTX vs. CDMARDs & $1.30(0.88,2.20)$ & $1.70(0.26,11.0)$ & $1.30(0.88,2.10)$ & 0.829 \\
\hline & ETN + MTX vs. ETN & $0.81(0.52,1.30)$ & $0.82(0.18,4.60)$ & $0.82(0.50,1.30)$ & 1.000 \\
\hline & $\mathrm{IFX}+\mathrm{MTX}$ vs. ABT + MTX & $2.40(0.96,6.60)$ & $1.00(0.56,2.00)$ & $1.30(0.82,2.40)$ & 0.145 \\
\hline & IFX + MTX vs. ETN + MTX & $1.30(0.50,3.80)$ & $1.30(0.34,4.90)$ & $1.30(0.61,3.30)$ & 0.989 \\
\hline & PBO vs. ADA & $1.00(0.62,1.70)$ & $0.90(0.16,5.00)$ & $1.00(0.61,1.50)$ & 0.875 \\
\hline & PBO vs. ETN & $0.61(0.07,4.30)$ & $0.82(0.31,2.10)$ & $0.76(0.28,1.70)$ & 0.780 \\
\hline & TCZ vs. cDMARDs & $1.70(0.81,3.50)$ & $1.40(0.55,4.40)$ & $1.60(0.93,2.80)$ & 0.839 \\
\hline & TCZ vs. PBO & $0.85(0.39,1.80)$ & $0.91(0.37,2.30)$ & $0.88(0.48,1.50)$ & 0.910 \\
\hline & TCZ + MTX vs. cDMARDs & $1.40(0.87,2.40)$ & $1.50(0.35,6.30)$ & $1.40(0.90,2.20)$ & 0.934 \\
\hline
\end{tabular}

PBO - placebo, MTX - methotrexate, IFX - infliximab, ETN - etanercept, ADA - adalimumab, GOL - golimumab, TCZ - tocilizumab, $A B T$ - abatacept, CZP - clonazepam, CDMARDs - traditional synthetic disease modifying antirheumatic drugs, ACR - American College of Rheumatology, AEs: adverse events, SAEs - serious adverse events. 
are shown in Table VII. As shown in Table VII, most scores are greater than 4 , which indicates that those included studies are of high quality.

\section{Discussion}

Based on the data and information of included RCTs, our study aims to evaluate the efficacy and safety of 15 drug therapies for RA patients. All available direct and indirect evidence of various treatment options was analyzed and compared simultaneously by NMA, which has a great advantage over traditional MA and makes up for the lack of head-to-head comparisons [80]. Therefore, our studies are much more reliable than the other MAs or NMAs. Moreover, it is more reasonable to select 4 efficacy and 2 safety endpoints as the evaluation indexes. Although there are also some NMA studies on this topic, they mostly include two or three outcomes to be compared. For example, some researchers only select ACR20 as the efficacy outcome and AEs as the safety outcome [1], which is not comprehensive enough.

After a systematic analysis of 15 therapies for patients with RA from 56 RCTs, we prefer to rec-

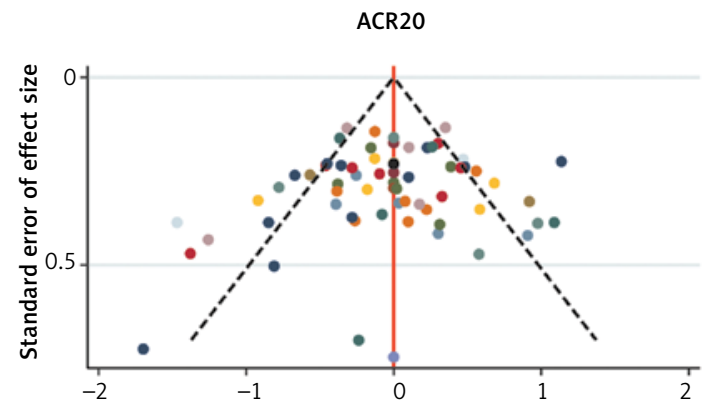

Effect size centred at comparison - specific pooled effect $\left(y_{i x y}-\mu_{x r}\right)$

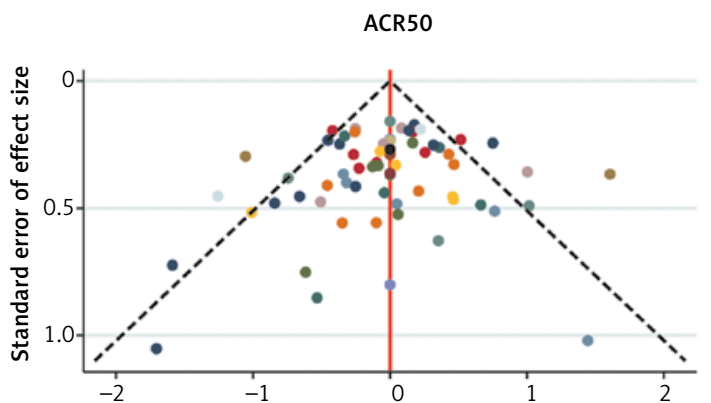

\begin{tabular}{|c|c|c|c|c|c|}
\hline $\begin{array}{l}\text { - J vs. K } \\
\text { A vs. I } \\
\text { D vs. K }\end{array}$ & $\begin{array}{l}\text { - J vs. O } \\
\text { B vs.J } \\
\text { D vs. N }\end{array}$ & $\begin{array}{l}\text { - L vs. O } \\
\text { - B vs. L } \\
\text { D vs. E }\end{array}$ & $\begin{array}{l}\text { - M vs. O } \\
\text { - B vs. O } \\
\text { - D vs. F }\end{array}$ & $\begin{array}{l}\text { - Avs. C } \\
\text { - C vs. D }\end{array}$ & $\begin{array}{l}\text { - Avs. D } \\
\text { - D vs.J }\end{array}$ \\
\hline
\end{tabular}

ACR70

Effect size centred at comparison - specific pooled effect $\left(y_{i x y}-\mu_{x y}\right)$

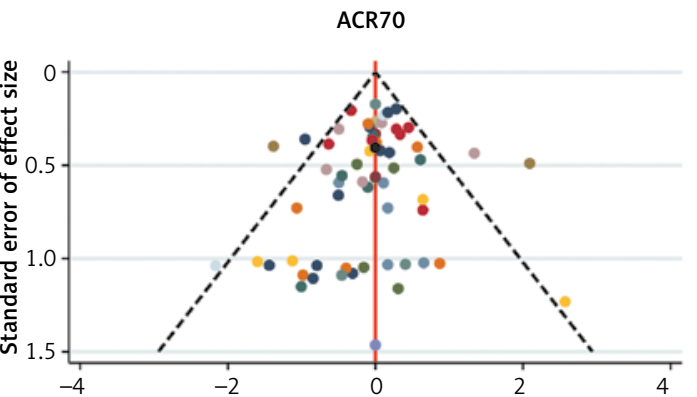

Effect size centred at comparison - specific pooled effect $\left(y_{i x y}-\mu_{x y}\right)$

\begin{tabular}{|llllll}
\hline J vs.K & $\bullet$ Jvs.O & $\bullet$ Lvs.O & $\bullet$ Mvs.O & $\bullet$ Avs.C & $\bullet$ Avs.D \\
$\bullet$ Avs.I & B Bvs.J & $\bullet$ Bvs.L & $\bullet$ Bvs.O & $\bullet$ C V v. D & $\bullet$ Dvs.J \\
$\bullet$ Dvs.K & $\bullet$ Dvs.N & Dvs.E & $\bullet$ Dvs.F & & \\
\hline
\end{tabular}

AE

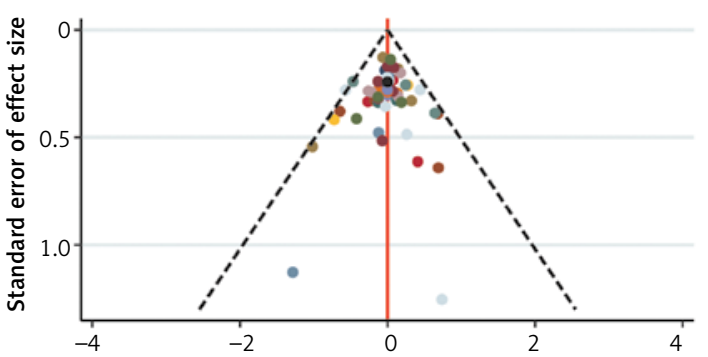

Effect size centred at comparison - specific pooled effect $\left(y_{i x y}-\mu_{x y}\right)$

\begin{tabular}{|llllll|}
$\bullet$ I vs. K & $\bullet$ J Jvs. O & $\bullet$ Lvs. O & $\bullet$ M vs. O & $\bullet$ A vs. C & $\bullet$ Avs. D \\
$\bullet$ Avs.I & $\bullet$ B vs.J & $\bullet$ B vs. L & $\bullet$ B vs. O & $\bullet$ C vs. D & $\bullet$ D vs.J \\
$\bullet$ D vs. K & $\bullet$ D vs. N & D Ds. E & $\bullet$ D vs. F & & \\
\hline
\end{tabular}

SAE

\begin{tabular}{|llllll|}
\hline I vs. K & $\bullet$ Jvs. O & $\bullet$ Lvs. O & $\bullet$ Mvs. O & $\bullet$ Avs. C & $\bullet$ A vs. D \\
$\bullet$ Avs.I & $\bullet$ B vs. L & $\bullet$ Bvs. O & $\bullet$ C vs. D & $\bullet$ Dvs.J & $\bullet$ D vs. K \\
$\bullet$ D vs. N & $\bullet$ D vs. E & $\bullet$ Dvs. F & $\bullet$ Dvs. G & & \\
\hline
\end{tabular}

Remission
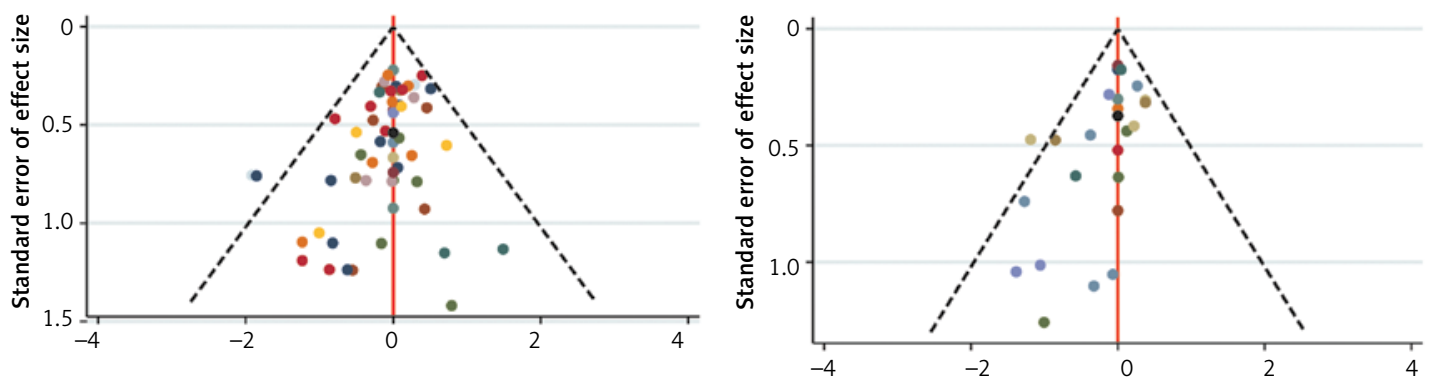

Effect size centred at comparison - specific pooled effect $\left(y_{i x y}-\mu_{x y}\right)$

\begin{tabular}{|c|c|c|c|c|c|}
\hline & $\begin{array}{l}\text { - J vs. O } \\
\text { B vs. L }\end{array}$ & $\begin{array}{l}- \text { L vs. O } \\
- \text { B vs. O }\end{array}$ & $\begin{array}{l}- \text { M vs. O } \\
\text { B vs. E }\end{array}$ & $\begin{array}{l}- \text { A vs. C } \\
- \text { C vs. D }\end{array}$ & $\begin{array}{l}\text { A v vs. } \mathrm{D} \\
\text { D D vs.J }\end{array}$ \\
\hline $\begin{array}{l}\text { A vs. I } \\
\text { D v vs. K }\end{array}$ & $\begin{array}{l}\text { B vs. L } \\
\text { D vs. N }\end{array}$ & $\begin{array}{l}\text { B vs. U } \\
\text { D vs. E }\end{array}$ & $\begin{array}{l}\text { B vs. E } \\
\bullet \text { D vs. F }\end{array}$ & & \\
\hline
\end{tabular}

Effect size centred at comparison - specific pooled effect $\left(y_{i x y}-\mu_{x y}\right)$

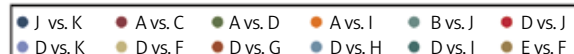
- Gvs. H

$A-A B T-M T X, B-A D A, C-A D A-M T X, D-C D M A R D S, E-E T N, F-E T N-M T X, G-G O L, H-G O L+M T X, I-I F X-M T X, J-T C Z$, $K-T C Z-M T X, L-A B T, M-C Z P, N-C Z P-M T X, O-P B O$

Figure 4. Publication bias of different outcomes 
Table VII. Jadad scale of 67 included studies

\begin{tabular}{|c|c|c|c|}
\hline Author, year & Randomized & Blinded & Withdrawal \\
\hline Abe et al., 2006 & 2 & 2 & 0 \\
\hline Bae et al., 2013 & 2 & 0 & 0 \\
\hline Kim et al., 2012 & 2 & 0 & 1 \\
\hline Combe et al., 2006 & 2 & 2 & 1 \\
\hline Combe et al., 2016 & 2 & 2 & 1 \\
\hline Dougados et al., 2013 & 2 & 2 & 1 \\
\hline Kameda et al., 2014 & 2 & 0 & 0 \\
\hline Jobanputra et al., 2012 & 2 & 0 & 1 \\
\hline Kay et al., 2008 & 2 & 2 & 0 \\
\hline Kim et al., 2007 & 2 & 2 & 1 \\
\hline Kremer et al., 2011 & 2 & 2 & 1 \\
\hline Kremer et al., 2006 & 2 & 2 & 0 \\
\hline Lan et al., 2004 & 2 & 2 & 0 \\
\hline Mathias et al., 2000 & 2 & 2 & 0 \\
\hline Moreland et al., 1999 & 2 & 2 & 1 \\
\hline Miyasaka et al., 2008 & 2 & 2 & 1 \\
\hline Nishimoto et al., 2009 & 2 & 2 & 1 \\
\hline O’Dell et al., 2013 & 2 & 2 & 1 \\
\hline O’Dell et al., 2012 & 2 & 2 & 1 \\
\hline Nishimoto et al., 2007 & 2 & 1 & 1 \\
\hline Tanake et al., 2016 & 2 & 2 & 0 \\
\hline Schiff et al., 2008 & 2 & 2 & 0 \\
\hline Van Riel et al., 2006 & 2 & 0 & 0 \\
\hline Van Riel et al., 2008 & 2 & 0 & 1 \\
\hline Van Vollenhoven et al., 2011 & 2 & 2 & 0 \\
\hline Weinblatt et al., 2003 & 2 & 2 & 1 \\
\hline Weinblatt et al., 2006 & 2 & 2 & 1 \\
\hline Westhovens et al., 2006 & 2 & 1 & 0 \\
\hline Zhang et al., 2006 & 2 & 2 & 1 \\
\hline Keystone et al., 2012 & 2 & 2 & 1 \\
\hline Smolen et al., 2009 & 2 & 2 & 1 \\
\hline Smolen et al., 2008 & 2 & 2 & 1 \\
\hline Gabay et al., 2012 & 2 & 2 & 0 \\
\hline Genovese et al., 2008 & 2 & 2 & 1 \\
\hline Yamamoto et al., 2014 & 2 & 2 & 1 \\
\hline Schiff et al., 2014 & 2 & 1 & 0 \\
\hline Weinblatt et al., 2012 & 2 & 2 & 1 \\
\hline Weinblatt et al., 2003 & 2 & 2 & 1 \\
\hline Fleischmann et al., 2012 & 2 & 2 & 1 \\
\hline Feischmann et al., 2009 & 2 & 2 & 1 \\
\hline Choy et al., 2012 & 2 & 2 & 1 \\
\hline
\end{tabular}


Table VII. Cont.

\begin{tabular}{|c|c|c|c|}
\hline Author, year & Randomized & Blinded & Withdrawal \\
\hline Keystone et al., 2009 & 2 & 2 & 0 \\
\hline Chen et al., 2009 & 2 & 2 & 0 \\
\hline Keystone et al., 2008 & 2 & 2 & 1 \\
\hline Maini et al., 2006 & 2 & 2 & 1 \\
\hline Maini et al., 1999 & 2 & 2 & 1 \\
\hline Vandeputte et al., 2004 & 2 & 2 & 1 \\
\hline Vandeputte et al., 2003 & 2 & 2 & 1 \\
\hline Klareskog et al., 2004 & 2 & 2 & 0 \\
\hline Lipsky et al., 2000 & 2 & 0 & 0 \\
\hline Kremer et al., 2003 & 2 & 2 & 0 \\
\hline Chen et al., 2016 & 2 & 2 & 1 \\
\hline Fleischmann et al., 2014 & 2 & 2 & 0 \\
\hline Machado et al., 2014 & 2 & 0 & 0 \\
\hline Kivitz et al., 2014 & 2 & 2 & 1 \\
\hline Kivitz et al., 2013 & 2 & 2 & 1 \\
\hline Hobbs et al., 2015 & 2 & 2 & 0 \\
\hline Li et al., 2015 & 2 & 2 & 1 \\
\hline Conaghan et al., 2013 & 2 & 2 & 1 \\
\hline Keystome et al., 2004 & 2 & 2 & 1 \\
\hline Defilippis et al., 2006 & 2 & 2 & 0 \\
\hline Weinbaltt et al., 2003 & 2 & 2 & 1 \\
\hline Weinbaltt et al., 1999 & 2 & 2 & 1 \\
\hline Smolen et al., 2011 & 2 & 2 & 1 \\
\hline Strand et al., 2011 & 2 & 2 & 1 \\
\hline Moreland et al., 1997 & 2 & 2 & 1 \\
\hline Furst et al., 2003 & 2 & 2 & 1 \\
\hline
\end{tabular}

Each question was to be answered with either a yes or a no. Each yes would score a single point, each no zero points. Additional points were given if: The method of randomization was described in the paper, and that method was appropriate (1 extra point in the randomization part); the method of blinding was described, and it was appropriate (1 extra point in the blinding part).

ommend CZP + MTX as the best treatment due to it having the highest rankings in ACR20 (83.3\%) and ACR50 (84.2\%) response rates and relatively low risk of adverse events. TCZ + MTX is recommended as an alternative treatment due to its good performance in all efficacy and safety outcomes. ABT is considered as the worst therapy, and CDMARDs is also not recommended though its safety seemed to be superior.

Interestingly, among these 15 drug therapies, six are biologics and another six are different combinations of MTX and biologics, including comparisons between $A B T$ and $A B T+M T X, A D A$ and ADA + MTX, CZP and CZP + MTX, ETN and ETN + MTX, $\mathrm{GOL}$ and $\mathrm{GOL}+\mathrm{MTX}$ as well as TCZ and TCZ + MTX.
It is easy to find that in most cases the efficacy and safety of a biological agent plus MTX are superior to the corresponding biologic agent alone. For example, the SUCRAs of efficacy and safety outcomes for ABT are as follows: 10.8\% (ACR20), 23.9\% (ACR50), 20\% (ACR70), 14.8\% (AEs) and $17.2 \%$ (SAEs). By contrast, ABT + MTX is more efficacious and safer with corresponding SUCRAs of $51 \%, 56.4 \%, 60.5 \%, 54.9 \%$ and $85.2 \%$. Previous researchers have also conducted direct comparisons between biologic monotherapy and a biological agent combined with MTX. For instance, Klareskog et al. demonstrated that the proportions of RA patients achieving ACR20, ACR50 and ACR70 were higher under the treatment of ETN + MTX than the 
ETN monotherapy. At week 52, about $85 \%, 69 \%$, $43 \%$ and $35 \%$ of patients in the ETN + MTX group achieved ACR20, ACR50, ACR70 and remission compared with $76 \%, 48 \%, 24 \%$ and $16 \%$ in the ETN groups [81], which is in line with our results.

However, a closer observation reveals that there is an exception. GOL + MTX performs better in all efficacy outcomes than GOL as the other treatments of a biologic agent plus MTX, while it performed worse in AEs and SAEs. There are also studies which presented a different conclusion. Some studies published before presented no difference between two kinds of treatment groups. Patients with RA treated with ETN and those treated with ETN + MTX were similar in ACR20, ACR50 and ACR70 (71.0\% vs. $67.1 \%, 41.9 \%$ vs. $40.1 \%$ and $17.4 \%$ vs. $18.4 \%$, respectively). The rates of adverse events and serious adverse events were also similar [82]. Maini et al. arrived at the same conclusion in the comparison between TCZ + MTX and TCZ [83]. Thus, further research should be conducted to estimate whether MTX benefits biologic monotherapy or not.

Although we have made the study as comprehensive as possible, there are still some limitations. Firstly, despite the fact that the inclusion trials were all RCTs, the results of efficacy and safety comparisons among 15 drug therapies still showed some statistical inconsistency. Perhaps the RCTs with contradictions between direct and indirect evidence should be reconsidered. Secondly, though disease durations of these interventions ranged from 14 weeks to 54 weeks, 16 of them only had a follow-up time of less than 20 weeks. A short duration is not enough to judge the safety of treatment [1]. Thirdly, medication dose, treatment cost, patient compliance and other influential factors also affected trial homogeneity. To some extent, the improvement in patients with RA is related to the dose of drugs, which was neglected in this study [83]. Last but not least, different RCTs included in our research had different definitions of safety outcomes. There is still a shortage of clear definition of AEs and SAEs.

In conclusion, we regard CZP + MTX as the optimal choice for RA patients in clinical practice and TCZ + MTX as an alternative treatment. Conversely, both $A B T$ and CDMARDs are not recommended. It is necessary to conduct long-term studies on patients with RA in order to provide a more complete assessment of diverse treatments and make a more judicious choice in clinical practice. In other words, we ought not only take into account clinical parameters such as ACR response rates and safety outcomes, but should also consider medication dose, treatment cost, patient compliance and so on. All efforts should be made to improve the life quality and health standard for patients with RA.

\section{Acknowledgments}

Kexun Ma and Ling Li are first co-authors.

This paper was supported by the National Natural Science Fund (81573869).

\section{Conflict of interest}

The authors declare no conflict of interest.

\section{References}

1. Lee YH, Bae SC. Comparative efficacy and safety of tocilizumab, rituximab, abatacept and tofacitinib in patients with active rheumatoid arthritis that inadequately responds to tumor necrosis factor inhibitors: a Bayesian network meta-analysis of randomized controlled trials. Int J Rheum Dis 2016; 19: 1103-11.

2. Donahue KE, Jonas DE, Hansen RA, et al. Drug Therapy for Rheumatoid Arthritis in Adults: An Update. Rockville (MD): Agency for Healthcare Research and Quality (US); 2012 Apr. PMID: 22696776.

3. Migliore A, Bizzi E, Egan CG, Bernardi M, Petrella L. Efficacy of biological agents administered as monotherapy in rheumatoid arthritis: a Bayesian mixed-treatment comparison analysis. Ther Clin Risk Manag 2015; 11: 1325-35.

4. Li R, Sun J, Ren LM, et al. Epidemiology of eight common rheumatic diseases in China: a large-scale cross-sectional survey in Beijing. Rheumatology (Oxford) 2012; 51: 721-9.

5. Singh JA, Hossain A, Tanjong Ghogomu E, et al. Biologics or tofacitinib for rheumatoid arthritis in incomplete responders to methotrexate or other traditional disease-modifying anti-rheumatic drugs: a systematic review and network meta-analysis. Cochrane Database Syst Rev 2016; 5: CD012183.

6. Fleischmann R, Vencovsky J, van Vollenhoven RF, et al. Efficacy and safety of certolizumab pegol monotherapy every 4 weeks in patients with rheumatoid arthritis failing previous disease-modifying antirheumatic therapy: the FAST4WARD study. Ann Rheum Dis 2009; 68: 805-11.

7. Graudal N, Hubeck-Graudal T, Tarp S, et al. Effect of combination therapy on joint destruction in rheumatoid arthritis: a network meta-analysis of randomized controlled trials. PLoS One 2014; 9: e106408.

8. Keystone EC, Genovese MC, Klareskog L, et al. Golimumab, a human antibody to tumour necrosis factor alpha given by monthly subcutaneous injections, in active rheumatoid arthritis despite methotrexate therapy: the GO-FORWARD Study. Ann Rheum Dis 2009; 68: 789-96.

9. Singh JA, Wells GA, Christensen R, et al. Adverse effects of biologics: a network meta-analysis and Cochrane overview. Cochrane Database Syst Rev 2011; 2: CD008794.

10. Lopez-Olivo MA, Tayar JH, Martinez-Lopez JA, et al. Risk of malignancies in patients with rheumatoid arthritis treated with biologic therapy: a meta-analysis. JAMA 2012; 308: 898-908.

11. Bongartz T, Sutton AJ, Sweeting MJ, et al. Anti-TNF antibody therapy in rheumatoid arthritis and the risk of serious infections and malignancies: systematic review and meta-analysis of rare harmful effects in randomized controlled trials. JAMA 2006; 295: 2275-85.

12. Lu G, Ades AE. Combination of direct and indirect evidence in mixed treatment comparisons. Stat Med 2004; 23: 3105-24. 
13. Chen XX, Li ZG, Wu HX, et al. A randomized, controlled trial of efficacy and safety of Anbainuo, a bio-similar etanercept, for moderate to severe rheumatoid arthritis inadequately responding to methotrexate. Clin Rheumatol 2016; 35: 2175-83.

14. Li Z, Zhang F, Kay J, et al. Efficacy and safety results from a phase 3, randomized, placebo-controlled trial of subcutaneous golimumab in Chinese patients with active rheumatoid arthritis despite methotrexate therapy. Int J Rheum Dis 2016; 11: 1143-56.

15. Hobbs K, Deodhar A, Wang B, et al. Randomized, double-blind, placebo-controlled study to evaluate the efficacy and safety of etanercept in patients with moderately active rheumatoid arthritis despite DMARD therapy. Springerplus 2015; 4: 113.

16. Yamamoto K, Takeuchi T, Yamanaka H, et al. Efficacy and safety of certolizumab pegol without methotrexate co-administration in Japanese patients with active rheumatoid arthritis: the HIKARI randomized, placebo-controlled trial. Mod Rheumatol 2014; 24: 552-60.

17. Weinblatt ME, Schiff MH, Valente R, et al. Head-to-head comparison of subcutaneous abatacept versus adalimumab on background methotrexate in rheumatoid arthritis: blinded two-year results from the ample study. Rheumatology (United Kingdom) 2014; 53: i88.

18. Schiff M, Weinblatt ME, Valente R, et al. Head-to-head comparison of subcutaneous abatacept versus adalimumab for rheumatoid arthritis: two-year efficacy and safety findings from AMPLE trial. Ann Rheum Dis 2014; 73: 86-94.

19. Machado DA, Guzman RM, Xavier RM, et al. Open-labe observation of addition of etanercept versus a conventional disease-modifying antirheumatic drug in subjects with active rheumatoid arthritis despite methotrexate therapy in the latin american region. J Clin Rheumatol 2014; 20: 25-33.

20. Kivitz A, Olech E, Borofsky $M$, et al. Subcutaneous tocilizumab versus placebo in combination with diseasemodifying antirheumatic drugs in patients with rheumatoid arthritis. Arthritis Care Res 2014; 66: 1653-61.

21. Fleischmann R, Koenig AS, Szumski A, et al. Short-term efficacy of etanercept plus methotrexate vs combinations of disease-modifying anti-rheumatic drugs with methotrexate in established rheumatoid arthritis. Rheumatology (Oxford) 2014; 53: 1984-93.

22. Weinblatt ME, Bingham lii CO, Mendelsohn AM, et al Intravenous golimumab is effective in patients with active rheumatoid arthritis despite methotrexate therapy with responses as early as week 2 : results of the phase 3 , randomised, multicentre, double-blind, placebo-controlled GO-FURTHER trial. Ann Rheum Dis 2013; 72: 381-9.

23. O'Dell JR, Mikuls TR, Taylor TH, et al. Therapies for active rheumatoid arthritis after methotrexate failure. N Eng J Med 2013; 369: 307-18.

24. Kivitz A, Olech E, Borofsky MA, et al. The safety and efficacy of tocilizumab subcutaneous in combination with traditional DMARDS in patients with moderate to severe rheumatoid arthritis up to 48 weeks (BREVACTA). Arthritis Rheum 2013; 65: S604-S5.

25. Gabay C, Emery P, Van Vollenhoven R, et al. Tocilizumab (TCZ) monotherapy is superior to adalimumab (ADA) monotherapy in reducing disease activity in patients with rheumatoid arthritis (RA): 24-week data from the phase 4 adacta trial. Ann Rheum Dis 2013; 71 (Suppl 3): 152.

26. Dougados M, Kissel K, Sheeran T, et al. Adding tocilizumab or switching to tocilizumab monotherapy in methotrexate inadequate responders: 24-week symp tomatic and structural results of a 2-year randomised controlled strategy trial in rheumatoid arthritis (ACTRAY). Ann Rheum Dis 2013; 72: 43-50.

27. Conaghan PG, Durez P, Alten RE, et al. Impact of intravenous abatacept on synovitis, osteitis and structural damage in patients with rheumatoid arthritis and an inadequate response to methotrexate: the ASSET randomised controlled trial. Ann Rheum Dis 2013; 72: 1287-94.

28. Bae SC, Gun SC, Mok CC, et al. Improved health outcomes with etanercept versus usual DMARD therapy in an Asian population with established rheumatoid arthritis. BMC Musculoskeletal Disord 2013; 14: 13.

29. Tanaka Y, Harigai M, Takeuchi T, et al. Golimumab in combination with methotrexate in Japanese patients with active rheumatoid arthritis: results of the GO-FORTH study. Ann Rheum Dis 2012; 71: 817-24.

30. O'Dell JR, Mikuls TR, Taylor T, et al. Rheumatoid arthritis comparison of active therapies in methotrexate suboptimal responders: validation of the strategy of conventional disease modifying anti-rheumatic drugs before biologicals. Arthr Rheum 2012; 64: S551-2.

31. Kim HY, Hsu PN, Barba M, et al. Randomized comparison of etanercept with usual therapy in an Asian population with active rheumatoid arthritis: the APPEAL trial. Int J Rheum Dis 2012; 15: 188-96.

32. Keystone EC, Wang MM, Layton $M$, et al. Clinical evaluation of the efficacy of the $\mathrm{P} 2 \mathrm{X} 7$ purinergic receptor antagonist AZD9056 on the signs and symptoms of rheumatoid arthritis in patients with active disease despite treatment with methotrexate or sulphasalazine. Ann Rheum Dis 2012; 71: 1630-5.

33. Jobanputra P, Maggs F, Deeming A, et al. Randomized efficacy and discontinuation study of etanercept versus adalimumab (Red sea): a pragmatic, open-label, non-inferiority study of first TNF inhibitor use in rheumatoid arthritis: 1-year data. Rheumatology 2012; 51: iii116-7.

34. Fleischmann R, Kremer J, Cush J, et al. Placebo-controlled trial of tofacitinib monotherapy in rheumatoid arthritis. N Engl J Med 2012; 367: 495-507.

35. Choy E, McKenna F, Vencovsky J, et al. Certolizumab pegol plus MTX administered every 4 weeks is effective in patients with RA who are partial responders to MTX. Rheumatology 2012; 51: 1226-34.

36. Van Vollenhoven RF, Kinnman N, Vincent E, et al. Atacicept in patients with rheumatoid arthritis and an inadequate response to methotrexate: results of a phase II, randomized, placebo-controlled trial. Arthritis Rheum 2011; 63: 1782-92.

37. Strand V, Smolen JS, Van Vollenhoven RF, et al. Certolizumab pegol plus methotrexate provides broad relief from the burden of rheumatoid arthritis: analysis of patient-reported outcomes from the RAPID 2 trial. Ann Rheum Dis 2011; 70: 996-1002.

38. Smolen J, Van Vollenhoven R, Kavanaugh A, et al. Efficacy and safety of certolizumab pegol plus methotrexate in patients with rheumatoid arthritis: 3-year data from the RAPID 2 study. J Rheum 2011; 38: 1152.

39. Kremer JM, Blanco R, Brzosko M, et al. Tocilizumab inhibits structural joint damage in rheumatoid arthritis patients with inadequate responses to methotrexate: results from the double-blind treatment phase of a randomized placebo-controlled trial of tocilizumab safety and prevention of structural joint damage at one year. Arthritis Rheum 2011; 63: 609-21.

40. Kameda H, Kanbe K, Sato E, et al. Etanercept (ETN) plus methotrexate (MTX) combination therapy resulted in 
a better radiographic outcome than ETN monotherapy even in patients with active rheumatoid arthritis despite MTX treatment: 104-week results from the JESMR study. Arthritis Rheum 2010; 62: 1812.

41. Smolen J, Landewé RB, Mease P, et al. Efficacy and safety of certolizumab pegol plus methotrexate in active rheumatoid arthritis: the RAPID 2 study. A randomised controlled trial. Ann Rheum Dis 2009; 68: 797-804.

42. Nishimoto N, Miyasaka N, Yamamoto K, et al. Study of active controlled tocilizumab monotherapy for rheumatoid arthritis patients with an inadequate response to methotrexate (SATORI): significant reduction in disease activity and serum vascular endothelial growth factor by IL- 6 receptor inhibition therapy. Mod Rheumatol 2009; 19: 12-9.

43. Keystone EC, Genovese MC, Klareskog L, et al. Golimumab, a human antibody to tumour necrosis factor alpha given by monthly subcutaneous injections, in active rheumatoid arthritis despite methotrexate therapy: the GO-FORWARD Study. Ann Rheum Dis 2009; 68: 789-96.

44. Fleischmann R, Vencovsky J, Van Vollenhoven RF, et al. Efficacy and safety of certolizumab pegol monotherapy every 4 weeks in patients with rheumatoid arthritis failing previous disease-modifying antirheumatic therapy: the FAST4WARD study. Ann Rheum Dis 2009; 68: 805-11.

45. Combe B, Codreanu C, Fiocco U, et al. Efficacy, safety and patient-reported outcomes of combination etanercept and sulfasalazine versus etanercept alone in patients with rheumatoid arthritis: a double-blind randomised 2-year study. Ann Rheum Dis 2009; 68: 1146-52.

46. Chen DY, Chou SJ, Hsieh TY, et al. Randomized, double-blind, placebo-controlled, comparative study of human anti-TNF antibody adalimumab in combination with methotrexate and methotrexate alone in Taiwanese patients with active rheumatoid arthritis. J Formos Med Assoc 2009; 108: 310-9.

47. van Riel PL, Freundlich B, MacPeek D, et al. Patient-reported health outcomes in a trial of etanercept monotherapy versus combination therapy with etanercept and methotrexate for rheumatoid arthritis: the ADORE trial. Ann Rheum Dis 2008; 67: 1104-10.

48. Smolen JS, Beaulieu A, Rubbert-Roth A, et al. Effect of interleukin-6 receptor inhibition with tocilizumab in patients with rheumatoid arthritis (OPTION study): a double-blind, placebo-controlled, randomised trial. Lancet 2008; 371: 987-97.

49. Schiff M, Keiserman M, Codding C, et al. Efficacy and safety of abatacept or infliximab vs placebo in ATTEST: a phase III, multi-centre, randomised, double-blind, placebo-controlled study in patients with rheumatoid arthritis and an inadequate response to methotrexate. Ann Rheum Dis 2008; 67: 1096-103.

50. Miyasaka N. Clinical investigation in highly disease-affected rheumatoid arthritis patients in Japan with adalimumab applying standard and general evaluation: the CHANGE study. Mod Rheumatol 2008; 18: 252-62.

51. Keystone E, Van Der Heijde D, Mason Jr D, et al. Certolizumab pegol plus methotrexate is significantly more effective than placebo plus methotrexate in active rheumatoid arthritis: findings of a fifty-two-week, phase III, multicenter, randomized, double-blind, placebo-controlled, parallel-group study. Arthritis Rheum 2008; 58: 3319-29.

52. Kay J, Matteson EL, Dasgupta B, et al. Golimumab in patients with active rheumatoid arthritis despite treatment with methotrexate: a randomized, double-blind, placebo-controlled, dose-ranging study. Arthritis Rheum 2008; 58: 964-75.
53. Genovese MC, MCKay JD, Nasonov EL, et al. Interleukin-6 receptor inhibition with tocilizumab reduces disease activity in rheumatoid arthritis with inadequate response to disease-modifying antirheumatic drugs: the tocilizumab in combination with traditional disease-modifying antirheumatic drug therapy study. Arthritis Rheum 2008; 58: 2968-80.

54. Nishimoto N, Hashimoto J, Miyasaka N, et al. Study of active controlled monotherapy used for rheumatoid arthritis, an IL-6 inhibitor (SAMURAI): evidence of clinical and radiographic benefit from an $X$ ray reader-blinded randomised controlled trial of tocilizumab. Ann Rheum Dis 2007; 66: 1162-7.

55. Kim HY, Lee SK, Song YW, et al. A randomized, double-blind, placebo-controlled, phase III study of the human anti-tumor necrosis factor antibody adalimumab administered as subcutaneous injections in Korean rheumatoid arthritis patients treated with methotrexate. APLAR J Rheumatol 2007; 10: 9-16.

56. Zhang FC, Hou Y, Huang F, et al. Infliximab versus placebo in rheumatoid arthritis patients receiving concomitant methotrexate: a preliminary study from China. APLAR J Rheumatol 2006; 9: 127-30.

57. Westhovens R, Yocum D, Han J, et al. The safety of infliximab, combined with background treatments, among patients with rheumatoid arthritis and various comorbidities: a large, randomized, placebo-controlled trial. Arthritis Rheum 2006; 54: 1075-86.

58. Weinblatt ME, Keystone EC, Furst DE, et al. Long term efficacy and safety of adalimumab plus methotrexate in patients with rheumatoid arthritis: ARMADA 4 year extended study. Ann Rheum Dis 2006; 65: 753-9.

59. Weinblatt $M$, Combe B, Covucci A, et al. Safety of the selective costimulation modulator abatacept in rheumatoid arthritis patients receiving background biologic and nonbiologic disease-modifying antirheumatic drugs: a one-year randomized, placebo-controlled study. Arthritis Rheum 2006; 54: 2807-16.

60. van Riel PL, Taggart AJ, Sany J, et al. Efficacy and safety of combination etanercept and methotrexate versus etanercept alone in patients with rheumatoid arthritis with an inadequate response to methotrexate: the ADORE study. Ann Rheum Dis 2006; 65: 1478-83.

61. Maini RN, Taylor PC, Szechinski J, et al. Double-blind randomized controlled clinical trial of the interleukin-6 receptor antagonist, tocilizumab, in European patients with rheumatoid arthritis who had an incomplete response to methotrexate. Arthritis Rheum 2006; 54: 2817-29.

62. Kremer JM, Genant HK, Moreland LW, et al. Effects of abatacept in patients with methotrexate-resistant active rheumatoid arthritis: a randomized trial. Ann Int Med 2006; 144: 865-76.

63. De Filippis L, Caliri A, Anghelone S, et al. Improving outcomes in tumour necrosis factor $\alpha$ treatment: comparison of the efficacy of the tumour necrosis factor alpha blocking agents etanercept and infliximab in patients with active rheumatoid arthritis. Panminerva Medica 2006; 48: 129-35.

64. Combe B, Codreanu C, Fiocco U, et al. Etanercept and sulfasalazine, alone and combined, in patients with active rheumatoid arthritis despite receiving sulfasalazine: a double-blind comparison. Ann Rheum Dis 2006; 65: 1357-62.

65. Abe T, Takeuchi T, Miyasaka N, et al. A multicenter, double-blind, randomized, placebo controlled trial of infliximab combined with low dose methotrexate in Japa- 
nese patients with rheumatoid arthritis. J Rheumatol 2006; 33: 37-44.

66. van de Putte LB, Atkins C, Malaise $M$, et al. Efficacy and safety of adalimumab as monotherapy in patients with rheumatoid arthritis for whom previous disease modifying antirheumatic drug treatment has failed. Ann Rheum Dis 2004; 63: 508-16.

67. Lan JL, Chou SJ, Chen DY, et al. A comparative study of etanercept plus methotrexate and methotrexate alone in Taiwanese patients with active rheumatoid arthritis: a 12-week, double-blind, randomized, placebo-controlled study. J Formos Med Assoc 2004; 103: 618-23.

68. Klareskog L, van der Heijde D, de Jager JP, et al. Therapeutic effect of the combination of etanercept and methotrexate compared with each treatment alone in patients with rheumatoid arthritis: double-blind randomised controlled trial. Lancet 2004; 363: 675-81.

69. Keystone EC, Kavanaugh AF, Sharp JT, et al. Radiographic, clinical, and functional outcomes of treatment with adalimumab (a human anti-tumor necrosis factor monoclonal antibody) in patients with active rheumatoid arthritis receiving concomitant methotrexate therapy: a randomized, placebo-controlled, 52-week trial. Arthritis Rheum 2004; 50: 1400-11.

70. Weinblatt ME, Keystone EC, Furst DE, et al. Adalimumab, a fully human anti-tumor necrosis factor alpha monoclonal antibody, for the treatment of rheumatoid arthritis in patients taking concomitant methotrexate: the ARMADA trial. Arthritis Rheum 2003; 48: 35-45.

71. van de Putte LB, Rau R, Breedveld FC, et al. Efficacy and safety of the fully human anti-tumour necrosis factor alpha monoclonal antibody adalimumab (D2E7) in DMARD refractory patients with rheumatoid arthritis: a 12 week, phase II study. Ann Rheum Dis 2003; 62: 1168-77.

72. Kremer JM, Westhovens R, Leon M, et al. Treatment of rheumatoid arthritis by selective inhibition of T-cell activation with fusion protein CTLA4Ig. N Eng J Med 2003; 349: 1907-15.

73. Furst DE, Schiff MH, Fleischmann RM, et al. Adalimumab, a fully human anti tumor necrosis factor-alpha monoclonal antibody, and concomitant standard antirheumatic therapy for the treatment of rheumatoid arthritis: results of STAR (Safety Trial of Adalimumab in Rheumatoid Arthritis). J Rheumatol 2003; 30: 2563-71.

74. Mathias SD, Colwell HH, Miller DP, et al. Health-related quality of life and functional status of patients with rheumatoid arthritis randomly assigned to receive etanercept or placebo. Clin Ther 2000; 22: 128-39.

75. Lipsky PE, van der Heijde DM, St Clair EW, et al. Infliximab and methotrexate in the treatment of rheumatoid arthritis. Anti-Tumor Necrosis Factor Trial in Rheumatoid Arthritis with Concomitant Therapy Study Group. N Engl J Med 2000; 343: 1594-602

76. Weinblatt ME, Kremer JM, Bankhurst AD, et al. A trial of etanercept, a recombinant tumor necrosis factor receptor:Fc fusion protein, in patients with rheumatoid arthritis receiving methotrexate. N Engl J Med 1999; 340: 253-9.

77. Moreland LW, Schiff MH, Baumgartner SW, et al. Etanercept therapy in rheumatoid arthritis: A randomized, controlled trial. Ann Intern Med 1999; 130: 478-86.

78. Maini R, St Clair EW, Breedveld F, et al. Infliximab (chimeric anti-tumour necrosis factor alpha monoclonal antibody) versus placebo in rheumatoid arthritis patients receiving concomitant methotrexate: a randomised phase III trial. Lancet 1999; 354: 1932-9.

79. Moreland LW, Baumgartner SW, Schiff MH, et al. Treatment of rheumatoid arthritis with a recombinant hu- man tumor necrosis factor receptor (p75)-Fc fusion protein. N Engl J Med 1997; 337: 141-7.

80. Jansen JP, Fleurence R, Devine B, et al. Interpreting indirect treatment comparisons and network meta-analysis for health-care decision making: report of the ISPOR Task Force on Indirect Treatment Comparisons Good Research Practices: part 1. Value Health 2011; 14: 417-28.

81. Klareskog L, van der Heijde D, de Jager JP, et al. Therapeutic effect of the combination of etanercept and methotrexate compared with each treatment alone in patients with rheumatoid arthritis: double-blind randomised controlled trial. Lancet 2004; 363: 675-81.

82. van Riel PL, Taggart AJ, Sany J, et al. Efficacy and safety of combination etanercept and methotrexate versus etanercept alone in patients with rheumatoid arthritis with an inadequate response to methotrexate: the ADORE study. Ann Rheum Dis 2006; 65: 1478-83.

83. Maini RN, Taylor PC, Szechinski J, et al. Double-blind randomized controlled clinical trial of the interleukin-6 receptor antagonist, tocilizumab, in European patients with rheumatoid arthritis who had an incomplete response to methotrexate. Arthritis Rheum 2006; 54: 2817-29. 